This version of astro-ph:0705.1315 contains the original (published) version of this article (Phys. Rev. D 76, 042008 (2007)), as well as its erratum. The original document has not been modified, but the reader should use the effective area tables from the erratum. 


\section{Multi-year search for a diffuse flux of muon neutrinos with AMANDA-II}

A. Achterberg, ${ }^{31}$ M. Ackermann, ${ }^{33}$ J. Adams, ${ }^{11}$ J. Ahrens, ${ }^{21}$ K. Andeen, ${ }^{20}$ J. Auffenberg, ${ }^{32}$ X. Bai, ${ }^{23}$ B. Baret ${ }^{9}$ S. W. Barwick,${ }^{16}$ R. Bay,${ }^{5}$ K. Beattie,${ }^{7}$ T. Becka, ${ }^{21}$ J. K. Becker, ${ }^{13}$ K.-H. Becker,${ }^{32}$ P. Berghaus, ${ }^{8,}{ }^{15}$ D. Berley, ${ }^{12}$ E. Bernardini, ${ }^{33}$ D. Bertrand, ${ }^{8}$ D. Z. Besson, ${ }^{17}$ E. Blaufuss, ${ }^{12}$ D. J. Boersma, ${ }^{20}$ C. Bohm,${ }^{27}$ J. Bolmont,${ }^{33}$ S. Böser,${ }^{33}$ O. Botner, ${ }^{30}$ A. Bouchta, ${ }^{30}$ J. Braun, ${ }^{20}$ T. Burgess, ${ }^{27}$ T. Castermans,${ }^{22}$ D. Chirkin, ${ }^{7}$ B. Christy, ${ }^{12}$ J. Clem, ${ }^{23}$ D. F. Cowen, ${ }^{29,} 28$ M. V. D'Agostino, ${ }^{5}$ A. Davour,${ }^{30}$ C. T. Day ${ }^{7}$ C. De Clercq,${ }^{9}$ L. Demirörs,${ }^{23}$ F. Descamps, ${ }^{14}$ P. Desiati, ${ }^{20}$ T. DeYoung,${ }^{29}$ J. C. Diaz-Velez, ${ }^{20}$ J. Dreyer, ${ }^{13}$ J. P. Dumm, ${ }^{20}$ M. R. Duvoort,${ }^{31}$ W. R. Edwards,${ }^{7}$ R. Ehrlich, ${ }^{12}$ J. Eisch, ${ }^{20}$ R. W. Ellsworth, ${ }^{12}$ P. A. Evenson, ${ }^{23}$ O. Fadiran, ${ }^{3}$ A. R. Fazely, ${ }^{4}$ K. Filimonov ${ }^{5}$ C. Finley, ${ }^{20}$ M. M. Foerster ${ }^{29}$ B. D. Fox,${ }^{29}$ A. Franckowiak, ${ }^{32}$ R. Franke, ${ }^{33}$ T. K. Gaisser, ${ }^{23}$ J. Gallagher, ${ }^{19}$ R. Ganugapati, ${ }^{20}$ H. Geenen, ${ }^{32}$ L. Gerhardt, ${ }^{16}$ A. Goldschmidt, ${ }^{7}$ J. A. Goodman, ${ }^{12}$ R. Gozzini, ${ }^{21}$ T. Griesel,${ }^{21}$ A. Groß, ${ }^{15}$ S. Grullon, ${ }^{20}$ R. M. Gunasingha, ${ }^{4}$ M. Gurtner,${ }^{32}$ C. Ha,${ }^{29}$ A. Hallgren,${ }^{30}$ F. Halzen, ${ }^{20}$ K. Han, ${ }^{11}$ K. Hanson, ${ }^{20}$ D. Hardtke, ${ }^{5}$ R. Hardtke, ${ }^{26}$ J. E. Hart, ${ }^{29}$ Y. Hasegawa, ${ }^{10}$ T. Hauschildt,${ }^{23}$ D. Hays, ${ }^{7}$ J. Heise, ${ }^{31}$ K. Helbing, ${ }^{32}$ M. Hellwig, ${ }^{21}$ P. Herquet, ${ }^{22}$ G. C. Hill,,${ }^{20}$ J. Hodges, ${ }^{20, *}$

K. D. Hoffman, ${ }^{12}$ B. Hommez, ${ }^{14}$ K. Hoshina, ${ }^{20}$ D. Hubert,${ }^{9}$ B. Hughey, ${ }^{20}$ J.-P. Hülß, ${ }^{1}$ P. O. Hulth, ${ }^{27}$ K. Hultqvist, ${ }^{27}$ S. Hundertmark,${ }^{27}$ M. Inaba, ${ }^{10}$ A. Ishihara, ${ }^{10}$ J. Jacobsen, ${ }^{7}$

G. S. Japaridze, ${ }^{3}$ H. Johansson, ${ }^{27}$ A. Jones, ${ }^{7}$ J. M. Joseph, ${ }^{7}$ K.-H. Kampert, ${ }^{32}$ A. Kappes ${ }^{20}{ }^{\dagger}$ †. Karg, ${ }^{32}$ A. Karle,${ }^{20}$ H. Kawai, ${ }^{10}$ J. L. Kelley ${ }^{20}$ F. Kislat,${ }^{6}$ N. Kitamura,${ }^{20}$ S. R. Klein, ${ }^{7}$ S. Klepser ${ }^{33}$ G. Kohnen, ${ }^{22}$ H. Kolanoski, ${ }^{6}$ L. Köpke,${ }^{21}$ M. Kowalski, ${ }^{6}$ T. Kowarik, ${ }^{21}$ M. Krasberg, ${ }^{20}$ K. Kuehn, ${ }^{16}$ M. Labare, ${ }^{8}$ H. Landsman, ${ }^{20}$ R. Lauer,${ }^{33}$ H. Leich, ${ }^{33}$ D. Leier, ${ }^{13}$ I. Liubarsky, ${ }^{18}$ J. Lundberg, ${ }^{30}$ J. Lünemann, ${ }^{13}$ J. Madsen, ${ }^{26}$ R. Maruyama, ${ }^{20}$ K. Mase,${ }^{10}$ H. S. Matis,${ }^{7}$ T. McCauley,${ }^{7}$ C. P. McParland,${ }^{7}$ A. Meli ${ }^{13}$ T. Messarius, ${ }^{13}$ P. Mészáros, ${ }^{28,} 29$ H. Miyamoto, ${ }^{10}$ A. Mokhtarani, ${ }^{7}$ T. Montaruli, ${ }^{20, \pm}$ A. Morey, ${ }^{5}$ R. Morse, ${ }^{20}$ S. M. Movit,${ }^{28}$ K. Münich, ${ }^{13}$ R. Nahnhauer, ${ }^{33}$ J. W. Nam, ${ }^{16}$ P. Nießen, ${ }^{23}$ D. R. Nygren,${ }^{7}$ H. Ögelman,${ }^{20}$ A. Olivas, ${ }^{12}$ S. Patton, ${ }^{7}$ C. Peña-Garay, ${ }^{25}$ C. Pérez de los Heros, ${ }^{30}$ A. Piegsa, ${ }^{21}$ D. Pieloth,${ }^{33}$ A. C. Pohl, ${ }^{30,}$ § R. Porrata, ${ }^{5}$ J. Pretz, ${ }^{12}$ P. B. Price,${ }^{5}$ G. T. Przybylski, ${ }^{7}$ K. Rawlins,${ }^{2}$ S. Razzaque, ${ }^{28,}{ }^{29}$ E. Resconi, ${ }^{15}$ W. Rhode,${ }^{13}$ 
M. Ribordy ${ }^{22}$ A. Rizzo, ${ }^{9}$ S. Robbins,${ }^{32}$ P. Roth, ${ }^{12}$ F. Rothmaier,${ }^{21}$ C. Rott,${ }^{29}$ D. Rutledge, ${ }^{29}$ D. Ryckbosch, ${ }^{14}$ H.-G. Sander, ${ }^{21}$ S. Sarkar,${ }^{24}$ K. Satalecka, ${ }^{33}$ S. Schlenstedt,${ }^{33}$ T. Schmidt, ${ }^{12}$ D. Schneider, ${ }^{20}$ D. Seckel, ${ }^{23}$ B. Semburg, ${ }^{32}$ S. H. Seo, ${ }^{29}$ Y. Sestayo, ${ }^{15}$ S. Seunarine, ${ }^{11}$ A. Silvestri, ${ }^{16}$ A. J. Smith, ${ }^{12}$ M. Solarz,${ }^{5}$ C. Song, ${ }^{20}$ J. E. Sopher,${ }^{7}$ G. M. Spiczak, ${ }^{26}$ C. Spiering, ${ }^{33}$ M. Stamatikos, ${ }^{20,}$ T. Stanev, ${ }^{23}$ T. Stezelberger, ${ }^{7}$ R. G. Stokstad, ${ }^{7}$ M. C. Stoufer ${ }^{7}$ S. Stoyanov ${ }^{23}$ E. A. Strahler, ${ }^{20}$ T. Straszheim, ${ }^{12}$ K.-H. Sulanke, ${ }^{33}$ G. W. Sullivan, ${ }^{12}$ T. J. Sumner, ${ }^{18}$ I. Taboada, ${ }^{5}$ O. Tarasova, ${ }^{33}$ A. Tepe, ${ }^{32}$ L. Thollander,${ }^{27}$ S. Tilav, ${ }^{23}$ M. Tluczykont,${ }^{33}$ P. A. Toale,${ }^{29}$ D. Tosi, ${ }^{33}$ D. Turčan, ${ }^{12}$ N. van Eijndhoven, ${ }^{31}$ J. Vandenbroucke, ${ }^{5}$ A. Van Overloop, ${ }^{14}$ V. Viscomi, ${ }^{29}$ B. Voigt, ${ }^{33}$ W. Wagner, ${ }^{29}$ C. Walck, ${ }^{27}$ H. Waldmann, ${ }^{33}$ M. Walter, ${ }^{33}$ Y.-R. Wang, ${ }^{20}$ C. Wendt, ${ }^{20}$ C. H. Wiebusch, ${ }^{1}$ C. Wiedemann, ${ }^{27}$ G. Wikström, ${ }^{27}$ D. R. Williams ${ }^{29}$ R. Wischnewski, ${ }^{33}$ H. Wissing, ${ }^{1}$ K. Woschnagg, ${ }^{5}$ X. W. Xu, ${ }^{4}$ G. Yodh, ${ }^{16}$ S. Yoshida, ${ }^{10}$ and J. D. Zornoza ${ }^{20, * *}$

(IceCube Collaboration)

${ }^{1}$ III Physikalisches Institut, RWTH Aachen University, D-52056 Aachen, Germany

${ }^{2}$ Dept. of Physics and Astronomy, University of Alaska Anchorage, 3211 Providence Dr., Anchorage, AK 99508, USA ${ }^{3}$ CTSPS, Clark-Atlanta University, Atlanta, GA 30314, USA

${ }^{4}$ Dept. of Physics, Southern University, Baton Rouge, LA 70813, USA

${ }^{5}$ Dept. of Physics, University of California, Berkeley, CA 94720, USA

${ }^{6}$ Institut für Physik, Humboldt-Universität zu Berlin, D-12489 Berlin, Germany

${ }^{7}$ Lawrence Berkeley National Laboratory, Berkeley, CA 94720, USA

${ }^{8}$ Université Libre de Bruxelles, Science Faculty CP230, B-1050 Brussels, Belgium

${ }^{9}$ Vrije Universiteit Brussel, Dienst ELEM, B-1050 Brussels, Belgium

${ }^{10}$ Dept. of Physics, Chiba University, Chiba 263-8522 Japan

${ }^{11}$ Dept. of Physics and Astronomy, University of Canterbury,

Private Bag 4800, Christchurch, New Zealand

${ }^{12}$ Dept. of Physics, University of Maryland, College Park, MD 20742, USA

${ }^{13}$ Dept. of Physics, Universität Dortmund, D-44221 Dortmund, Germany

${ }^{14}$ Dept. of Subatomic and Radiation Physics, University of Gent, B-9000 Gent, Belgium 
${ }^{15}$ Max-Planck-Institut für Kernphysik, D-69177 Heidelberg, Germany ${ }^{16}$ Dept. of Physics and Astronomy,

University of California, Irvine, CA 92697, USA

${ }^{17}$ Dept. of Physics and Astronomy, University of Kansas, Lawrence, KS 66045, USA

${ }^{18}$ Blackett Laboratory, Imperial College, London $S W 72 B W, U K$

${ }^{19}$ Dept. of Astronomy, University of Wisconsin, Madison, WI 53706, USA

${ }^{20}$ Dept. of Physics, University of Wisconsin, Madison, WI 53706, USA

${ }^{21}$ Institute of Physics, University of Mainz,

Staudinger Weg 7, D-55099 Mainz, Germany

${ }^{22}$ University of Mons-Hainaut, 7000 Mons, Belgium

${ }^{23}$ Bartol Research Institute and Department of Physics and Astronomy, University of Delaware, Newark, DE 19716, USA

${ }^{24}$ Dept. of Physics, University of Oxford, 1 Keble Road, Oxford OX1 3NP, UK

${ }^{25}$ Institute for Advanced Study, Princeton, NJ 08540, USA

${ }^{26}$ Dept. of Physics, University of Wisconsin, River Falls, WI 54022, USA

${ }^{27}$ Dept. of Physics, Stockholm University, SE-10691 Stockholm, Sweden

${ }^{28}$ Dept. of Astronomy and Astrophysics,

Pennsylvania State University, University Park, PA 16802, USA

${ }^{29}$ Dept. of Physics, Pennsylvania State University, University Park, PA 16802, USA

${ }^{30}$ Division of High Energy Physics, Uppsala University, S-75121 Uppsala, Sweden

${ }^{31}$ Dept. of Physics and Astronomy, Utrecht University/SRON, NL-3584 CC Utrecht, The Netherlands

${ }^{32}$ Dept. of Physics, University of Wuppertal, D-42119 Wuppertal, Germany ${ }^{33}$ DESY, D-15735 Zeuthen, Germany 


\begin{abstract}
A search for $\mathrm{TeV}-\mathrm{PeV}$ muon neutrinos from unresolved sources was performed on AMANDA-II data collected between 2000 and 2003 with an equivalent livetime of 807 days. This diffuse analysis sought to find an extraterrestrial neutrino flux from sources with non-thermal components. The signal is expected to have a harder spectrum than the atmospheric muon and neutrino backgrounds. Since no excess of events was seen in the data over the expected background, an upper limit of $E^{2} \Phi_{90 \% \text { C.L. }}<7.4 \times 10^{-8} \mathrm{GeV} \mathrm{cm}^{-2} \mathrm{~s}^{-1} \mathrm{sr}^{-1}$ is placed on the diffuse flux of muon neutrinos with a $\Phi \propto \mathrm{E}^{-2}$ spectrum in the energy range $16 \mathrm{TeV}$ to $2.5 \mathrm{PeV}$. This is currently the most sensitive $\Phi \propto \mathrm{E}^{-2}$ diffuse astrophysical neutrino limit. We also set upper limits for astrophysical and prompt neutrino models, all of which have spectra different than $\Phi \propto \mathrm{E}^{-2}$.
\end{abstract}

PACS numbers: 95.55.Vj, 95.75.-z, 95.85.Ry

${ }^{*}$ Corresponding author: hodges@icecube.wisc.edu (J. Hodges)

$\dagger^{\dagger}$ on leave of absence from Universität Erlangen-Nürnberg, Physikalisches Institut, D-91058, Erlangen,

Germany

$\ddagger_{\text {}}$ on leave of absence from Università di Bari, Dipartimento di Fisica, I-70126, Bari, Italy

$\S$ affiliated with Dept. of Chemistry and Biomedical Sciences, Kalmar University, S-39182 Kalmar, Sweden

『NASA Goddard Space Flight Center, Greenbelt, MD 20771, USA

**affiliated with IFIC (CSIC-Universitat de València), A. C. 22085, 46071 Valencia, Spain 


\section{INTRODUCTION}

High energy photons have been used to paint a picture of the non-thermal Universe, but a more complete image of the hot and dense regions of space can potentially be obtained by combining astrophysical neutrino and gamma ray data. Neutrinos can provide valuable information because they are undeflected by magnetic fields and hence their paths point back to the particle's source. Unlike photons, neutrinos are only rarely absorbed when traveling through matter. However, their low interaction cross section also makes their detection more challenging. The observation of astrophysical neutrinos would confirm the predictions $[1,2$, 3, 4, 5, 6, 7, 8, 9] that neutrinos are produced in hadronic interactions in cosmic accelerators, such as active galactic nuclei or gamma-ray bursts.

Instead of searching for neutrinos from either a specific time or location in the sky, this analysis searches for extraterrestrial neutrinos from unresolved sources. If the neutrino flux from an individual source is too small to be detected by point source search techniques, it is nevertheless possible that many sources, isotropically distributed throughout the Universe, could combine to make a detectable signal. An excess of events over the expected atmospheric neutrino background would be indicative of an extraterrestrial neutrino flux.

In this paper, we report on a search for a diffuse flux of astrophysical muon neutrinos performed with data collected by the AMANDA-II neutrino telescope from 2000 - 2003. To perform the search, a 5.2 sr sky region (slightly less than $2 \pi$ sr) was monitored over a four year period, for a total of 807 days of livetime. Before describing specifics of the analysis, the existing diffuse neutrino models and limits and how we aim to detect neutrinos are described in Sections [I] and [II. In Section [V], typical backgrounds to the extraterrestrial signal are discussed, as well as how events are simulated in the detector. We also explain how an atmospheric neutrino sample was obtained. An extensive systematic uncertainty study is described in Section V, The relationship between up and downgoing events is explored in Section VI. Finally, the results of the analysis are presented in Section VII. Since no excess of high energy events was seen above the predicted atmospheric neutrino background, we set limits on the flux of extraterrestrial muon neutrinos with a generic $\Phi \propto \mathrm{E}^{-2}$ energy spectrum as well as with a number of different model spectra discussed in Section VIIB. 


\section{ASTROPHYSICAL FLUXES AND LIMITS}

The analysis presented in this paper assumes a $\Phi \propto \mathrm{E}^{-2}$ spectrum resulting from shock acceleration processes. Although other spectra were tested, the $\Phi \propto \mathrm{E}^{-2}$ spectral shape is considered a benchmark to characterize acceleration in many sources.

The Waxman-Bahcall upper bound [1, 2, 3] follows an $\Phi \propto \mathrm{E}^{-2}$ spectrum and reaching below the sensitivity of this bound has traditionally been a goal of neutrino experiments. Nellen, Mannheim and Biermann [4] and Becker, Biermann and Rhode [5] have suggested $\Phi \propto \mathrm{E}^{-2}$ neutrino spectra with higher normalizations than the Waxman-Bahcall bound. The other astrophysical neutrino models tested here (Mannheim, Protheroe and Rachen [6], Stecker, Done, Salamon and Sommers [7, 8] and Loeb and Waxman [9]) predict different spectral shapes and are specific predictions of neutrino fluxes from classes of objects such as active galactic nuclei (AGN) and starburst galaxies. The models have been derived based on a variety of astronomical results, including the observed extragalactic cosmic ray flux and $\mathrm{x}$-ray and radio measurements.

A precursor to this muon neutrino analysis was conducted with data collected in 1997 by the AMANDA-B10 detector [10]. (In 1997, the AMANDA detector consisted of 10 sensor strings, a subset of the 19 strings in the final AMANDA-II configuration.) Other AMANDA analyses have focused on the search for a diffuse flux of neutrinos using particle showers or cascades [11]. Cascades are caused by $\nu_{e}$ and $\nu_{\tau}$ charged current interactions and all-flavor neutral current interactions in the ice near the detector. Even though no extraterrestrial signal has been detected, models can be excluded by setting upper limits.

The Fréjus [12], MACRO [13] and Baikal [14]) experiments have set upper limits on the flux of astrophysical neutrinos in the same energy region as this analysis (TeV - PeV). Published upper limits from these experiments assuming a $\Phi \propto \mathrm{E}^{-2}$ spectrum are summarized along with the results of this analysis in Section VIIA Depending on the detector and the specific analysis, the reported upper limit constrains either the muon neutrino flux or the all-flavor neutrino flux. Upper limits obtained from all-flavor analyses are not directly comparable to $\nu_{\mu}$ upper limits. However, for a wide range of neutrino production models and oscillation parameters, the flavor flux ratio at Earth can be approximated as 1:1:1 [15]. In that case, either a single-flavor limit can be multiplied by three and compared to an allflavor result, or an all-flavor limit can be divided by three and compared to a single-flavor 
result.

The Baikal experiment has placed limits on models with spectra other than $\Phi \propto E^{-2}[14]$, which are compared to the results from this analysis in Section VIIB. Here, nine different spectral shapes are tested, including the search for prompt neutrinos from the decay of charmed particles. Since this analysis is optimized on energy-dependent parameters, the optimization was performed individually for each energy spectrum.

\section{NEUTRINO DETECTION IN AMANDA}

Although chargeless particles like neutrinos are not directly observable, the by-products of their interactions with polar ice or rock near the detector can be observed. In particular, two types of neutrino-induced events can be distinguished in AMANDA. All neutrino flavors can cause hadronic or electromagnetic showers in the ice and these appear as a momentary point-like source of Cherenkov light. Alternatively, long track-like events are the signature of neutrino-induced muons traveling through the detector. A cone of Cherenkov light is emitted by these muons as they travel faster than the speed of light in ice. The present analysis focuses exclusively on the muon track channel for identifying neutrino events. Tau neutrinos can undergo charged current interactions and contribute to the upgoing $\mu$ and $\nu_{\mu}$ fluxes via $\tau \rightarrow \mu \nu_{\tau} \bar{\nu}_{\mu}$ decay. Although $\nu_{\tau}$ interactions and $\tau$ decay may contribute between $10 \%$ to $16 \%$ to the $\mathrm{E}^{-2}$ signal flux [16], this contribution is ignored in this analysis.

Nineteen vertical strings hold the optical modules (OMs) for recording the timing and position of detected photons, which is needed for reconstructing the path of the muon [17]. The angular distribution between the neutrino direction and the reconstructed muon track has a median of $2^{\circ}$ when the highest quality events are used. The 677 OMs each consist of a photomultiplier tube (PMT) enclosed in a pressure-resistant glass sphere. The OMs are deployed to depths between 1500 and 2000 meters. An event is recorded when at least 24 OMs report seeing light within a $2.5 \mu$ s window. AMANDA has been operating in the final configuration with 19 strings (AMANDA-II) since 2000 [17]. 


\section{SEARCH METHODS}

This analysis uses the Earth as a filter to search for upgoing astrophysical neutrinoinduced events. The background for the analysis consists of atmospheric muons and neutrinos created when cosmic rays interact with Earth's atmosphere. The majority of the events registered in the detector are atmospheric muons traveling downward through the ice.

Conventional atmospheric neutrinos arise from the decay of pions and kaons created in cosmic ray interactions with the atmosphere. Atmospheric neutrinos are able to travel undisturbed through the Earth. They can be separated from atmospheric muons by their direction, namely by demanding that the reconstructed track is upgoing. The conventional atmospheric neutrino flux asymptotically approaches a $\Phi \sim \mathrm{E}^{-3.7}$ spectrum in the multi$\mathrm{TeV}$ range. Prompt neutrinos are the counterpart of the conventional atmospheric neutrino flux and will be discussed in Section VII.

In the initial sample of $5.2 \times 10^{9}$ events, many downgoing events were misreconstructed as upgoing tracks. Misreconstruction happens because photons scatter in the ice, causing directional and timing information to be lost. Hence, the selected upgoing event sample not only contains truly upgoing neutrinos, but a certain fraction of downgoing atmospheric muons.

An energy-correlated observable was used to separate neutrino-induced events since the predicted astrophysical neutrino flux has a much harder energy spectrum $\left(\Phi \propto \mathrm{E}^{-2}\right)$ than the conventional atmospheric neutrinos from pions and kaons. Any excess of events at high energy over the expected atmospheric neutrino background indicates the presence of a signal.

The search method can be summarized by the following three selection steps:

1. Use the zenith angle from the reconstructed track to reject obviously downgoing events.

2. Select events that have observables more consistent with typical long upgoing tracks. This separates truly upgoing events from misreconstructed downgoing events.

3. Use an energy-related observable (number of OMs triggered) to separate upgoing atmospheric neutrinos from upgoing astrophysical neutrinos.

This analysis relied on simulated data sets of background and signal events. Sixty-three days of downgoing atmospheric muons were simulated with CORSIKA [18] version 6.030 and the QGSJET01 hadronic interaction model. The events were simulated with a $\Phi \propto \mathrm{E}^{-2.7}$ 
primary energy spectrum. These downgoing events are so frequent $(\sim 80 \mathrm{~Hz}$ at trigger level) that two atmospheric muon events produced by unrelated primaries often occur in the detector during the same detector trigger window of $2.5 \mu \mathrm{s}$. Timing patterns of the light from the two tracks may be such that the reconstruction results in a single upgoing track. These coincident muon events may be caused by two muons which are each individually incapable of triggering the detector with at least $24 \mathrm{OM}$ hits. However, events which only hit a few OMs can now trigger the detector when in coincidence with another event. This means that a simple trigger rate calculation of $80 \mathrm{~Hz} \times 80 \mathrm{~Hz} \times 2.5 \mu$ s is not possible since all combinations of events with a total of at least 24 hits can trigger the detector. These coincident muon events were simulated for 826 days of livetime and have a frequency of about $\sim 2-3 \mathrm{~Hz}$ at trigger level.

Muon neutrinos with a $\Phi \propto \mathrm{E}^{-1}$ spectrum were simulated with nusim [19] and reweighted to atmospheric neutrino flux predictions [20, 21, 22, 23, 24], as well as to an astrophysical muon neutrino flux of $\mathrm{E}^{2} \Phi=1 \times 10^{-6} \mathrm{GeV} \mathrm{cm}^{-2} \mathrm{~s}^{-1} \mathrm{sr}^{-1}$. The normalization of the test signal spectrum, which is irrelevant when setting a limit, was taken to be approximately equal the previous upper limit from the AMANDA-B10 diffuse analysis [10].

\section{A. Filtering the Data Set}

The 2000 - 2003 analysis covers 807 days of detector livetime between February 2000 and November 2003. Because of summer maintenance operations, no data were used from the polar summer seasons. In the first stage of the analysis, reconstruction software was used to make an initial hypothesis on the track direction of every event based on the timing pattern of the detected light [17].

Figure 1 shows the zenith angle of the reconstructed tracks for all events at the beginning of the analysis (level 0 ). Vertically downgoing tracks have a reconstructed zenith angle of $0^{\circ}$ $(\cos (\theta)=1)$. The data set was reduced to $8 \times 10^{6}$ events by removing all events reconstructed with zenith angles less than $80^{\circ}(\cos (\theta)=0.17)$. The remaining data set mainly consists of misreconstructed downgoing muons and events near the horizon.

The reduction of the data by three orders of magnitude with the simple zenith requirement made it feasible to perform more CPU-intensive track reconstructions on the remaining events. Track parameters were adjusted to maximize the log likelihood, given the observed 


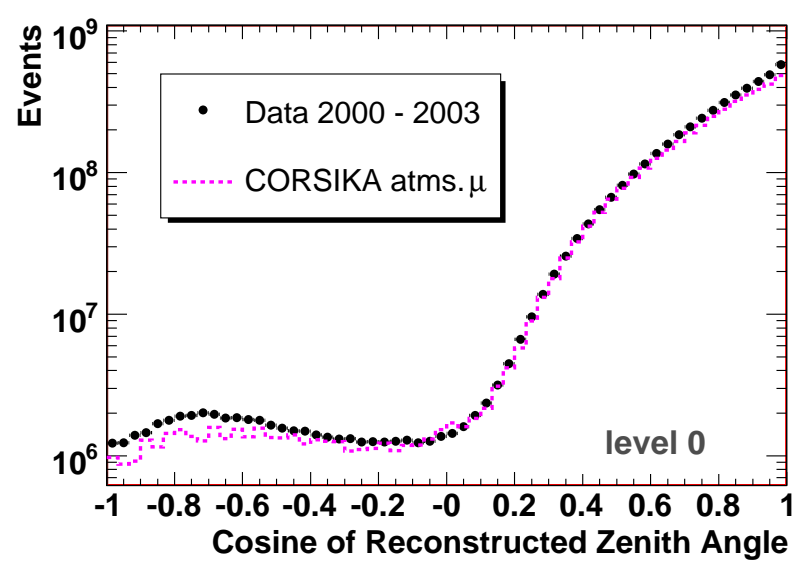

FIG. 1: The cosine of the reconstructed zenith angle is shown for every event at the beginning of the analysis (level 0). The experimental data is dominated by downgoing atmospheric muons. Events reconstructed as upgoing appear on the left side of the plot and downgoing events appear on the right.

light pattern. Many of the Cherenkov photons scatter multiple times as they travel through the ice and this changes their direction and delays the times at which they are likely to be detected. An iterative technique was performed in which each event was reconstructed 32 times [17], each time with a different seed. Each iteration shifts the zenith and azimuth of the track and moves the track to pass through the center of gravity of the hits. The best track found by the iterative search was used throughout the later stages of the analysis.

In order to prevent any inadvertent tuning of the event selection criteria that would bias the result, a blindness procedure was followed which required that further event selections were developed only on simulation and low energy data, where the signal is negligible compared to the background. The number of OMs triggered (from now on indicated by $N_{\mathrm{ch}}$, or number of channels hit) is the energy-correlated observable used to separate atmospheric neutrinos from astrophysical ones (Figure 2). Only low energy data events (low $N_{\text {ch }}$ values) were compared to simulation. High energy data events (high $N_{\text {ch }}$ values) were only revealed once the final event selection was established. Energy and $N_{\mathrm{ch}}$ are correlated since high energy events release more energy in the detector causing more hits than low energy ones. However, the correlation is not perfect since high energy events occurring far from the detector may trigger only a few OMs.

Event selection was based on observables associated with the reconstructed tracks [17] and 


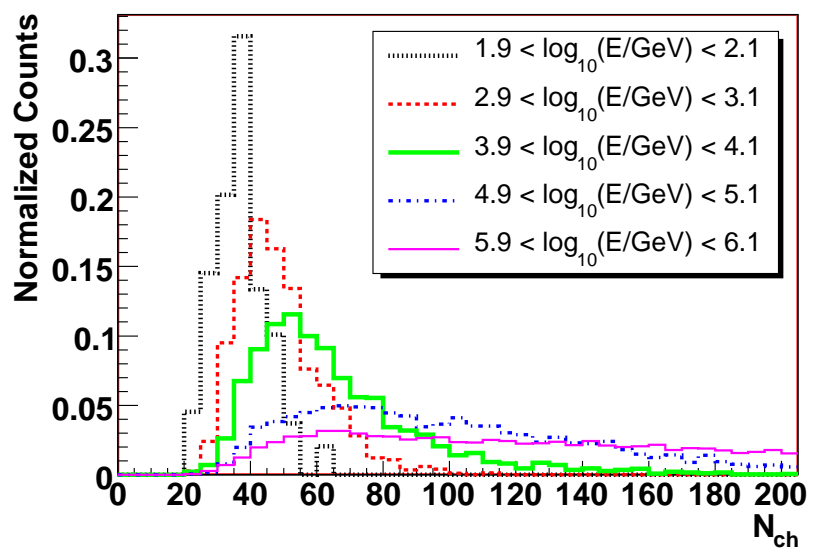

FIG. 2: The number of OMs hit during an event $\left(N_{\mathrm{ch}}\right)$ was used as an energy-correlated observable. Each line on this $N_{\mathrm{ch}}$ distribution represents events with approximately the same simulated energy. High energy events may not be contained within the detector and hence can trigger a wide $N_{\mathrm{ch}}$ span.

is described in more detail in Appendix A. In order to separate misreconstructed downgoing events and coincident muons from the atmospheric and astrophysical neutrinos, events were required to have observables consistent with long tracks and many photons with arrival times close to those predicted for un-scattered propagation. The number of photons arriving between -15 and +75 ns of their expected un-scattered photon arrival time is referred to as the number of direct hits $\left(N_{\text {dir }}\right)$. The direct length $\left(L_{\text {dir }}\right)$ is the maximum separation of two direct hits along the reconstructed track. The smoothness $(S)$ is a measurement of how uniformly all hits are distributed along the track and it varies between -1.0 and 1.0. Positive values of the smoothness indicate more hits at the beginning of a track and negative values indicate more hits occur toward the end. Evenly distributed hits will have smoothness values near 0 . The median resolution $(M R)$ is calculated from a paraboloid fit to the likelihood minimum for the track [25]. This method analyzes the angular resolution on an event-by-event basis. Lastly, high quality events have higher values of the logarithm of the up-to-down likelihood ratio, $\Delta L=\left(-\log \mathcal{L}_{\text {down }}\right)-\left(-\log \mathcal{L}_{\text {up }}\right)$. The likelihoods $\mathcal{L}_{\text {up }}$ and $\mathcal{L}_{\text {down }}$ are the product of the values of the probability density function for the observed photon arrival times, for the best upgoing and zenith-weighted downgoing track reconstruction [17], respectively. A more strict requirement for the likelihood ratio was applied to vertical events than for events near the horizon. Horizontal events tend to have smaller likelihood ratios 
since the zenith angle difference between the best upgoing and zenith-weighted downgoing track hypothesis is often small.

The event selection requirements were successively tightened, based on the reconstructed track parameters, establishing five quality levels. At Level 5, used for the final stages of the analysis, the event sample is expected to contain only truly upgoing events. The zenith angle distribution for the events at each quality level is shown in Figure 3, Although the entire upgoing zenith angle region is being studied, the event selection requirements preferentially retain vertically upgoing events. Horizontal and vertical events must pass the same requirements for track length and number of direct hits, however this is more difficult for horizontal events since the detector is not as wide as it is tall.

\section{B. Separating Atmospheric Neutrinos from Astrophysical Neutrinos}

Figure 4 shows the $N_{\mathrm{ch}}$ distribution for events at Level 5 . The optimal place for the energy-correlated event observable requirement was established with the simulation by minimizing the expected Model Rejection Factor (MRF) [26]. The Feldman-Cousins method was used to calculate the median upper limit [27]. The MRF is the median upper limit divided by the number of predicted signal events for the $\nu_{\mu}$ signal being tested. The MRF was calculated for every possible $N_{\text {ch }}$ value and was at its minimum at $N_{\text {ch }} \geq 100$. Hence, the optimal separation of astrophysical and atmospheric neutrinos is achieved with this $N_{\mathrm{ch}}$ requirement.

The final event sample was composed of events which pass all event selection requirements (Level 5) and have $N_{\mathrm{ch}} \geq 100$. After the high $N_{\mathrm{ch}}$ requirement, the atmospheric neutrino simulation peaked at $10 \mathrm{TeV}$, while the signal simulation peaked around $100 \mathrm{TeV}$ (Figure 5). The energy range defined by the central $90 \%$ of the signal with $N_{\mathrm{ch}} \geq 100$ is the energy range for the sensitivity or limit. For this search, the central $90 \%$ signal region extends from $16 \mathrm{TeV}$ to $2.5 \mathrm{PeV}$.

The efficiency of the detector for neutrinos is quantified by the effective area. In the energy range relevant to this analysis, it increases with energy and is further enhanced by including uncontained events. The effective area is described by the following equation where $N$ represents the number of observed events and $T$ is the detector livetime: 

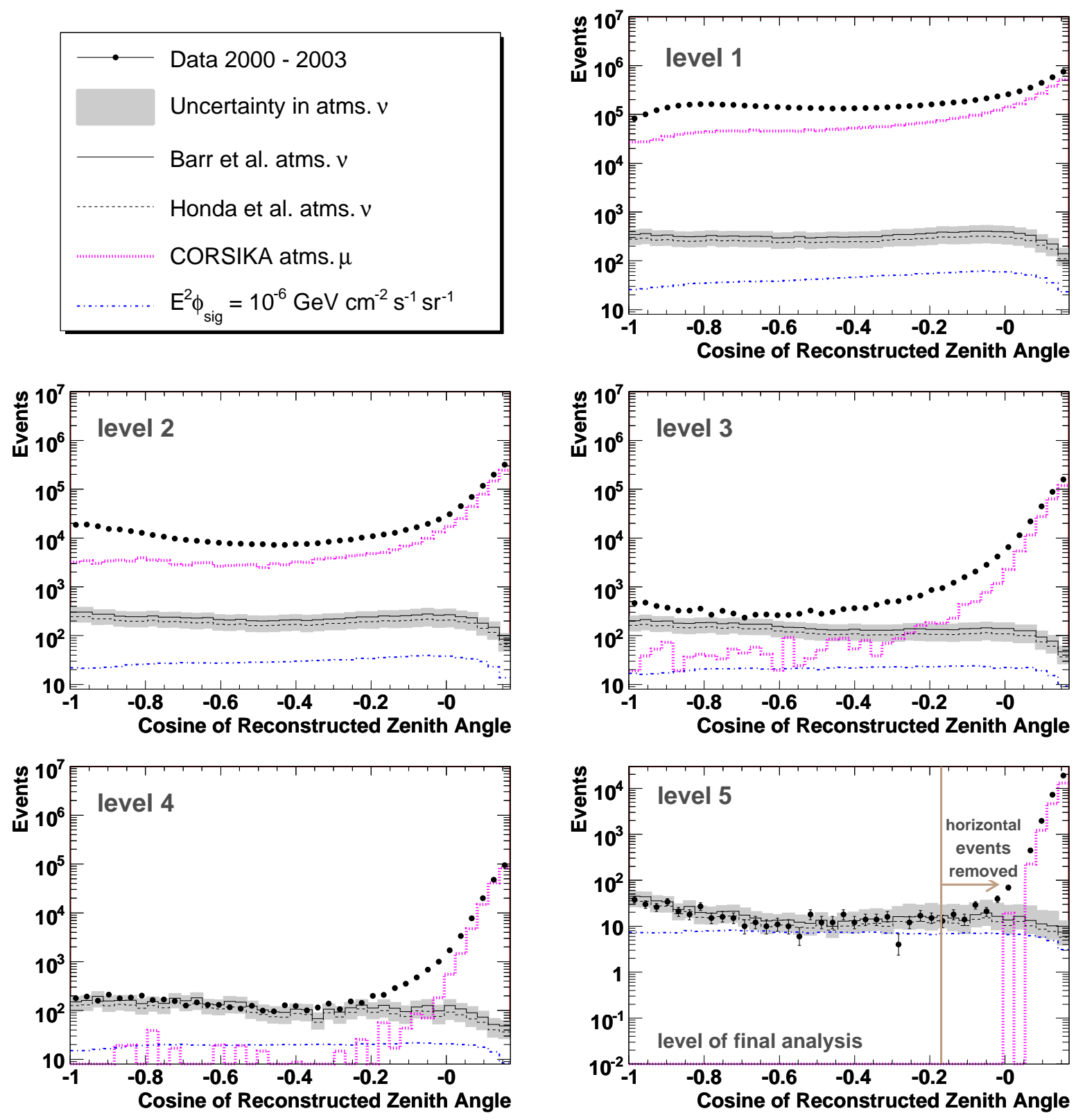

FIG. 3: The cosine of the zenith angle is plotted for all events surviving the event quality criteria at a given level. Events at $\cos ($ zenith $)=-1$ are traveling straight up through the detector from the Northern Hemisphere. The initial zenith angle requirement removed events from $0^{\circ}$ to $80^{\circ}$ (level 1 - top right). Events reconstructed just above the horizon appear at the right side of each plot. Each level represents an increasingly tighter set of quality requirements. As the quality level increased, misreconstructed downgoing muons were eliminated. To ensure a clean upgoing sample, events coming from the horizon were discarded by requiring reconstruction angles greater than $100^{\circ}$. The final analysis was performed at level 5 (bottom right) with horizontal events removed. 


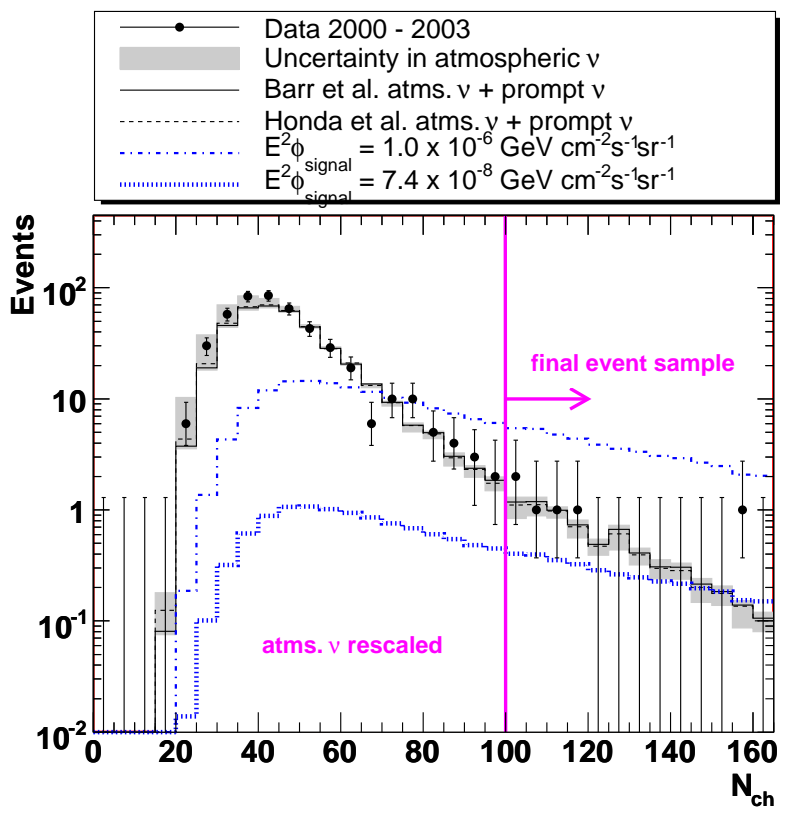

FIG. 4: $N_{\mathrm{ch}}$, or number of OMs hit. Prediction for both conventional and prompt atmospheric neutrinos are shown and their uncertainties are represented by the gray band. The central prompt neutrino flux used here is the average of the Martin GBW [30] and Naumov RQPM [31, 32] models. All atmospheric neutrinos are normalized to the number of data events in the range $50<N_{\mathrm{ch}}<100$. The lower signal flux curve corresponds to the limit obtained in this paper.

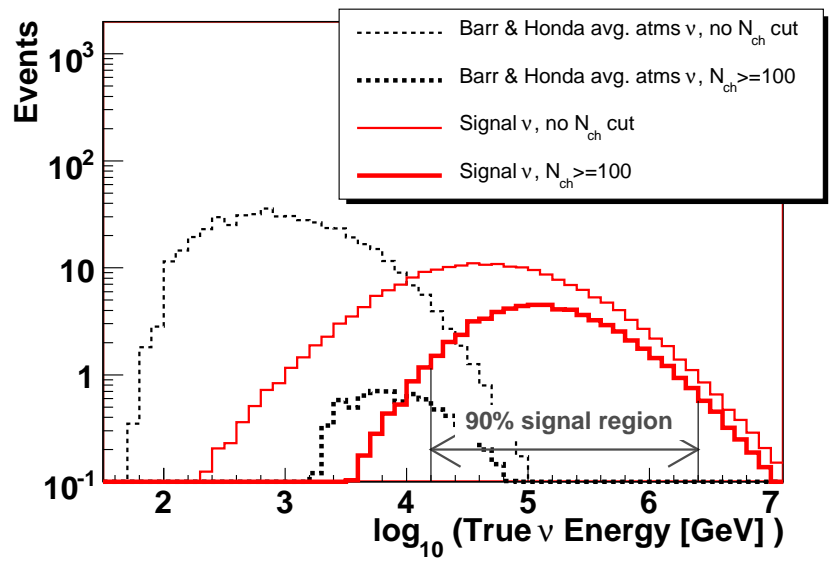

FIG. 5: The true energy of the simulation is shown for atmospheric neutrino and signal events. The thin dashed (atms. $\nu$ ) and solid (signal $\nu$ ) curves represent the number of events before the $N_{\mathrm{ch}} \geq 100$ requirement. The thick dashed and solid lines represent only the events in the high energy sample. 


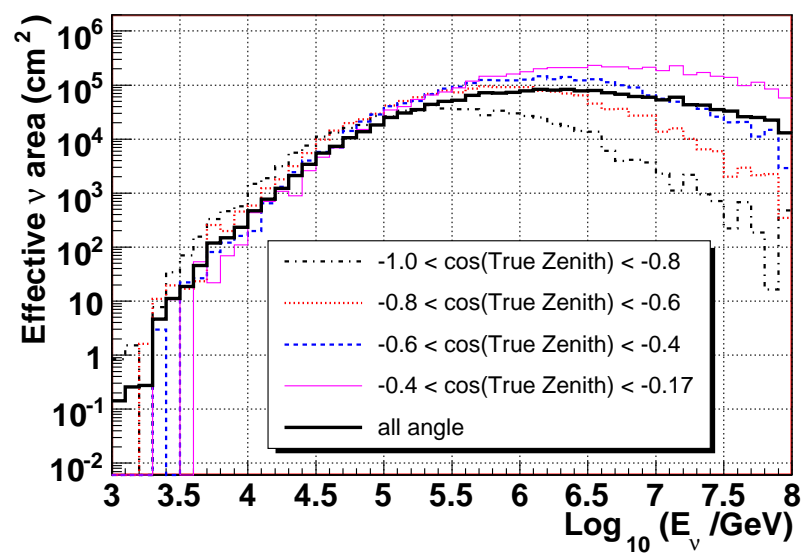

FIG. 6: Effective area for $\nu_{\mu}$ as a function of the true simulated energy at the Earth's surface in intervals of cosine of the true zenith angle. The effective area is the equivalent area over which the detector would be $100 \%$ efficient for detecting neutrinos. The absorption of neutrinos and reduction of their energy via neutral current interactions in the Earth are taken into account. The angle-averaged effective area is represented by the solid black line.

$$
\frac{N}{T}=\int A_{\text {eff }}^{\nu}\left(E_{\nu}, \Omega\right) \Phi_{\nu} d \Omega d E
$$

The effective area as a function of energy is shown for different zenith angle regions in Figure 6 (and is tabulated in Appendix B). At energies greater than $10^{5} \mathrm{GeV}$, the Earth begins to be opaque to neutrinos depending on direction and the highest energy events are most likely to come from the region around the horizon [28]. In Figure 6, the effective area decreases at high energy because tracks with zenith angles between $80^{\circ}$ and $100^{\circ}$ were discarded. Most of the events that were removed were high energy events from the horizon.

\section{SYSTEMATIC UNCERTAINTIES}

A discovery is made if an excess of events over the predicted background is observed in the data. However, due to uncertainties in the simulation, the number of signal and background events predicted may not accurately reflect the true signal and background. Theoretical uncertainties exist in the atmospheric neutrino flux models for several reasons. The cosmic ray spectrum is very uncertain at high energy and hadronic interactions in this energy range are not well understood. There are also detector-related uncertainties. Photons scatter more 
in dirty or bubble-laden ice. Hence, our incomplete understanding of the dust layers in the ice and the bubbles in the hole ice (formed from water that refroze after deployment of the OMs) add uncertainty to our models [29]. There are also uncertainties in the simulation associated with the modeling of light propagation in the ice and with the optical module sensitivity. These contributions are considered individually to see how they affect the number of simulated events in the final sample. The number of experimental data events remaining after the final energy requirement $\left(N_{\mathrm{ch}} \geq 100\right)$ is then compared to the range of predicted background and signal events when uncertainties are considered.

\section{A. Theoretical Uncertainty in the Background}

For this analysis, two models based on the work of Barr et al. [21, 22, 23] and Honda et al. [20] were considered equally likely options for the background atmospheric neutrino simulation. These two models are recent calculations that cover the highest and lowest portion of the atmospheric neutrino flux band created by uncertainties in the primary cosmic ray flux and the high energy hadronic interaction models. Since these models do not extend to the high energies needed for this analysis, the models were extrapolated to higher energies based on the procedure described in Appendix C. Differences between the Barr et al. and Honda et al. models are also summarized there.

Conventional atmospheric neutrinos from the decay of pions and kaons are not the only source of atmospheric background. Above $50 \mathrm{TeV}-1 \mathrm{PeV}$, the source of atmospheric neutrinos is expected to change [30, 31, 32, 33, 34]. Semileptonic decays of short-lived charmed particles become the main contributor to the atmospheric neutrino flux. Since these charmed particles decay quickly before they can lose much energy, the resulting neutrinos are called prompt neutrinos. At these energies, charm quarks are produced primarily via gluongluon fusion. Uncertainties in the gluon distribution at low Bjorken $x$ lead to uncertainties in this prompt lepton flux.

Uncertainties were included for both conventional atmospheric neutrino models. The uncertainty in the cosmic ray spectrum was estimated as a function of energy based on the spread of values measured by many cosmic ray experiments [35]. These uncertainties were added in quadrature with the estimated uncertainty due to choosing different hadronic

interaction models [20, 21, 36]. Uncertainties were also estimated based on the spread of 
predictions surrounding the unknown prompt neutrino flux. Unless mentioned otherwise, when prompt neutrinos were included in this work, the average of the Martin GBW (GolecBiernat and Wüsthoff) [30] and Naumov RQPM (Recombination Quark Parton Model) [31, 32] models is shown. This is henceforth called the central prompt neutrino model.

All of the uncertainty factors for the total (conventional + prompt) atmospheric neutrino flux were combined and are shown as a function of energy in Appendix C. Since the true energy of every simulated event is known, each event was given a weight based on the maximum uncertainty estimated for that neutrino energy. As a result, three predictions for the number of atmospheric neutrinos in the final high energy sample were made (the model, the model plus maximum energy-dependent uncertainty, the model minus maximum energy-dependent uncertainty). Since both the Barr et al. and Honda et al. fluxes were considered equally likely, the central prompt neutrino flux was added to both predictions. Then uncertainties were added and subtracted to both of these total atmospheric neutrino fluxes, creating six different background possibilities.

\section{Normalizing the Atmospheric Neutrino Simulation to the Data}

After all but the $N_{\mathrm{ch}}$ event selection requirements were fulfilled, the $N_{\mathrm{ch}}$ distribution for the observed low energy events was inconsistent with that for the total atmospheric neutrino simulation in normalization. Each of the six atmospheric neutrino background predictions was renormalized to match the number of data events observed in the low $N_{\text {ch }}$ region, where the signal was insignificant compared to the background. By rescaling the simulation to the number of observed data events, the uncertainty in the atmospheric neutrino flux was reduced to the uncertainty in the spectral shape.

Since some of the atmospheric neutrino models predicted more events than the data while others predicted less, renormalization of the models to the data brought the simulated models into closer agreement. The renormalization is explained in greater detail in Appendix C.

Since the purpose of this normalization was to correct for theoretical uncertainties in the atmospheric neutrino background prediction, it was not applied to the simulated neutrino signal. 


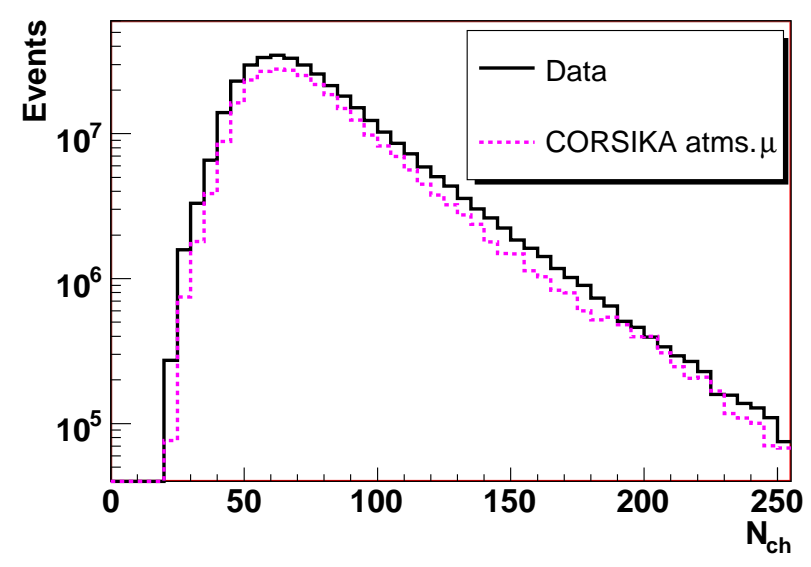

FIG. 7: In the inverted analysis, the highest quality downgoing events were studied. The $N_{\mathrm{ch}}$ distribution is shown for all events which survive the inverted quality requirements.

\section{B. Simulation Uncertainties}

To assure that the detector response to high energy events $\left(N_{\mathrm{ch}} \geq 100\right)$ is understood, it is important to study energetic events while simultaneously keeping the high energy upgoing events blind to the analyzer. To this end, an inverted analysis was performed in which high quality downgoing tracks were selected from the initial data set. The advantage of studying high quality downgoing tracks is that large data sets are available to study both the high and low energy events. When the data and simulation observable distributions are not perfectly matched, imposing event quality requirements may result in removing different fractions of the simulation in comparison with the data. The inverted analysis was used to study this systematic effect.

\section{Inverted Analysis Using Atmospheric Muons}

For the inverted analysis, all event quality requirements described previously (and summarized in Appendix A) were applied, but events were selected based on a high probability of being downgoing rather than upgoing tracks.

When compared to the downgoing experimental data, small shifts were observed in the peaks of the simulated distributions for the number of direct hits $\left(N_{\text {dir }}\right)$, the smooth distribution of hits along the track $(S)$, the event-by-event track resolution $(M R)$ and likelihood of being downgoing muon tracks rather than upgoing (Inverted Likelihood Ratio, ILR). These 
discrepancies are most likely due to inaccurate modeling of optical ice properties in the simulation, since it is technically challenging to implement a detailed description of photon propagation through layered ice.

If multiple parameters are correlated, it is possible that mismatches in one parameter may affect the agreement between data and simulation in another. In order to study these effects, the differences in the data and simulation were analyzed at the level where no quality criteria had been applied. The simulated distributions needed to be shifted to larger values by approximately $10 \%$ for $N_{\text {dir }}, 8 \%$ for $S, 5 \%$ for $M R$ and $1 \%$ for $I L R$. When simultaneous corrections to the simulation for all of these effects were applied, the downgoing data and simulation were in better agreement for all parameter distributions. Later in the analysis, these shifts were applied to the upgoing analysis. The number of background and signal events appearing in the final upgoing sample was recalculated based on these simulation shifts.

\section{Uncertainties in Detector Response}

The downgoing sample from the inverted analysis was also used to study how well the

detector response was simulated in the high energy $\left(N_{\mathrm{ch}} \geq 100\right)$ regime. Using downgoing data and atmospheric muon simulation, a ratio of the number of events was taken as a function of $N_{\mathrm{ch}}$ from the histograms shown in Figure 7 . If the simulation perfectly described the data, the shapes of the $N_{\mathrm{ch}}$ distributions would match and this ratio would be flat. The downgoing ratio was mostly flat, but slightly increased at large $N_{\text {ch }}$ where low statistics introduced large uncertainties. The statistical uncertainty aside, a scenario was considered in which the downgoing data to simulation ratio truly increased as $N_{\text {ch }}$ increased. Under this scenario, the simulation is renormalized by a larger factor at high $N_{\text {ch }}$ to replicate the data. This $N_{\mathrm{ch}}$-dependent renormalization was then applied to the upgoing simulation used for the main part of the analysis. This non-linear normalization factor had a negligible effect in the number of atmospheric neutrinos predicted in the final sample of events with $N_{\text {ch }} \geq 100$. However, the high energy signal simulation event rate increased by $25 \%$ when this non-linear $N_{\text {ch }}$ effect was included. This uncertainty was incorporated in the final limit calculation that will be described in the next section.

Detection efficiency also depends on the OM sensitivity. This parameter of the simulation 
was modified and new simulated events were generated. After comparing the data and simulation with different OM sensitivities, a $10 \%$ uncertainty in the total number of events due to inaccurate modelling of the OM detection sensitivity was incorporated into the final upper limit calculation.

The systematic errors due to the neutrino interaction cross-section, rock density (below the detector), and muon energy loss do not contribute significantly to this analysis [16].

\section{RELATIONSHIP BETWEEN UPGOING AND DOWNGOING EVENTS}

In addition to using the inverted analysis to study high energy events and the bias introduced by inaccurate simulation, the downgoing events can be used as a calibration beam for the upgoing atmospheric neutrino flux. To do this, the same simulation package (CORSIKA v6.030, QGSJET01, $\Phi_{\text {primary }} \propto \mathrm{E}^{-2.7}$ ) was used to describe the downgoing atmospheric muons and the upgoing atmospheric neutrinos [37].

As shown in Table \, the ratio of experimental data to CORSIKA downgoing muon simulation was relatively constant as the event selection became more discriminating. The simulation does not match the data normalization and this may be a consequence of the theoretical imperfections in the CORSIKA simulation (mainly due to the hadronic interaction model (QGSJET01) and uncertainty in the primary spectrum $\left.\left(\Phi \propto \mathrm{E}^{-2.7}\right)\right)$. Another contributing factor to the normalization difference may be that light propagation in the layered ice is modeled inaccurately. When the upgoing CORSIKA atmospheric neutrinos are rescaled based on the downgoing muons, then the upgoing experimental data and CORSIKA atmospheric neutrino simulation are in good agreement for the number of low energy events in the final sample. This can only be seen when the tightest criteria are applied because misreconstructed muons and coincident muons contaminate the data sample when the quality requirements are loose. For instance, at level 5 in the inverted analysis, the ratio of downgoing data to simulation was 1.22. For the upgoing analysis at level 5, 146 events were observed and 124.9 CORSIKA atmospheric neutrinos were predicted. When adjusted based on the inverted analysis, $152.4(=124.9 \times 1.22)$ CORSIKA atmospheric neutrinos were predicted, which is in good agreement with the observed value. This shows that it is possible to adjust the normalization of the upgoing events based on the downgoing observations (when the up and downgoing simulation use the same input assumptions about the spectrum and 


\begin{tabular}{|l|l|l|l|l|l|}
\hline & L1 & L2 & L3 & L4 & L ${ }^{*}$ \\
\hline Downgoing & & & & & \\
\hline data $\left(\times 10^{8}\right)$ & 7.88 & 6.70 & 6.05 & 5.89 & 2.59 \\
\hline $\begin{array}{l}\text { CORSIKA } \\
\text { atms. } \mu\left(\times 10^{8}\right)\end{array}$ & 6.63 & 5.75 & 5.12 & 5.01 & 2.12 \\
\hline ratio & 1.19 & 1.17 & 1.18 & 1.18 & 1.22 \\
\hline Upgoing & & & & & \\
\hline signal & 325 & 241 & 191 & 185 & 103 \\
\hline coinc $\mu$ & 2570 & 268 & 45.8 & 29.4 & 0 \\
\hline $\begin{array}{l}\text { misreconstructed } \\
\text { CORSIKA } \\
\text { atms. } \mu\end{array}$ & & & & & \\
\hline $\begin{array}{l}\text { Barr } \text { et al. } \\
\text { atms. } \nu\end{array}$ & 37800 & 2570 & 148 & 34.2 & 0 \\
\hline $\begin{array}{l}\text { Honda } \text { et al. } \\
\text { atms. } \nu\end{array}$ & 681 & 526 & 393 & 380 & 194 \\
\hline Martin GBW prompt $\nu$ & 1.9 & 1.9 & 1.6 & 1.5 & 0.7 \\
\hline Naumov RQPM prompt $\nu$ & 18.9 & 18.9 & 16.0 & 15.5 & 7.5 \\
\hline $\begin{array}{l}\text { CORSIKA } \\
\text { atms. } \nu\end{array}$ & 440 & 335 & 251 & 243 & 125 \\
\hline $\begin{array}{l}\text { Adjusted } \\
\text { CORSIKA } \\
\text { atms. } \nu\end{array}$ & 524 & 392 & 296 & 286 & 152 \\
\hline data & 276894 & 24422 & 1269 & 531 & 146 \\
\hline
\end{tabular}

TABLE I: The number of low energy events $\left(50<N_{\mathrm{ch}}<100\right)$ at a given level (see Appendix A) for the different types of simulation and experimental data. The top portion of the table presents results from the inverted analysis. The main upgoing analysis is summarized in the lower portion of the table. Note that the upgoing data and adjusted CORSIKA atmospheric neutrino flux are in good agreement when the CORSIKA neutrino events are adjusted by the scale factor determined in the downgoing analysis. This agreement can be seen at the tightest quality levels because all misreconstructed backgrounds have been removed.

interaction model).

\section{RESULTS}

We calculated a confidence interval based on the number of events in the final $N_{\mathrm{ch}} \geq 100$ sample of the predicted background and signal and the observed data. Statistical and systematic uncertainties were incorporated into the confidence interval such that the true, 


\begin{tabular}{|l|c|}
\hline \multicolumn{2}{|l|}{ Systematic Uncertainty } \\
\hline Theoretical uncertainty in atms. $\nu$ flux & See Figure 12 \\
\hline Number of Direct Hits & $10 \%$ \\
\hline Smoothness & $8 \%$ \\
\hline Median Resolution & $5 \%$ \\
\hline Inverted Likelihood Ratio & $1 \%$ \\
\hline Total background uncertainty & $\mathbf{+ 1 9 \%} / \mathbf{- 1 8 \%}$ \\
\hline \hline Non-linear detector response at high $N_{\mathrm{ch}}$ & $25 \%$ \\
\hline OM sensitivity & $10 \%$ \\
\hline Total signal efficiency uncertainty & $+/-\mathbf{2 7 \%}$ \\
\hline
\end{tabular}

TABLE II: The systematic error was estimated with several techniques. The theoretical uncertainty in the atmospheric neutrino flux was estimated as a function of energy (Section VA). Using the inverted analysis, shifts were observed between the data and simulation in four parameters (Section VB 1). When each of the above mentioned uncertainty factors was applied to the atmospheric neutrino simulation, the resulting spread in the number of events predicted in the $N_{\mathrm{ch}} \geq 100$ sample indicated that the total background uncertainty was $+19 \% /-18 \%$ of the average predicted background, 7.0 events. The non-linear response of the detector in $N_{\text {ch }}$ was estimated as $25 \%$ (Section VB 2). When added in quadrature with the 10\% uncertainty in OM sensitivity (Section VB 2), the total signal efficiency uncertainty was $+/-27 \%$.

but unknown, value of the diffuse flux of astrophysical neutrinos is contained within the interval in $90 \%$ of repeated experiments. A hybrid frequentist-Bayesian method based on the work of Cousins and Highland [38] was used to construct a confidence belt with systematic uncertainties. The likelihood ratio ordering was based on the unified confidence intervals explained by Feldman and Cousins [27]. The uncertainty in the detection efficiency of the signal was set at 27\% (10\% for optical module sensitivity added in quadrature with $25 \%$ for non-linearity in the $N_{\mathrm{ch}}$ spectrum when data and simulation are compared). Systematic uncertainties on the number of background events in the final sample were also included in the confidence belt construction. Inclusion of the signal and background uncertainties followed the methods described by Conrad et al. [39] and Hill [40].

In constructing the flat Bayesian prior for the background, twelve atmospheric neutrino 
models were considered equally likely. The twelve predictions were derived as follows. Initially, two background predictions were considered, Barr et al. and Honda et al., each with the central prompt neutrino flux added. To include systematic uncertainties in the models, maximum uncertainties were added and subtracted from each model (Section VA $)$. Hence, the six predictions were named Barr et al. maximum, nominal and minimum and Honda et al. maximum, nominal and minimum. The number of events predicted for the background in the final sample is listed in Appendix C. To account for systematic uncertainties in the detector response, the simulation was shifted in four different parameters as described in Section VB 1. This simulation shift was performed on each of the 6 models described above, hence creating a total of 12 different atmospheric neutrino predictions that were used in the confidence belt construction. The number of events predicted by the 6 models with shifted simulation was within $10 \%$ of each number reported in Appendix C.

\section{A. Results for $\Phi \propto \mathbf{E}^{-2}$}

The signal hypothesis consisted of a flux $\mathrm{E}^{2} \Phi=1.0 \times 10^{-6} \mathrm{GeV} \mathrm{cm}^{-2} \mathrm{~s}^{-1} \mathrm{sr}^{-1}$. At this signal strength, 66.7 signal events were expected in the final $N_{\mathrm{ch}} \geq 100$ data. (This value assumes half of the correction from the simulation shifts since 68.4 events were predicted in the final selection, but the number of events decreased to 65.0 when the simulation shifts were applied.) The sensitivity was obtained from the slice of the confidence belt corresponding to zero signal strength. The median observation assuming no signal was seven events, giving a median event upper limit of 6.36 and hence a sensitivity of $9.5 \times 10^{-8} \mathrm{GeV} \mathrm{cm}^{-2} \mathrm{~s}^{-1} \mathrm{sr}^{-1}$.

When the data with $N_{\text {ch }} \geq 100$ were revealed, six data events were observed. This was consistent with the average expected atmospheric neutrino background of 7.0 events (after averaging all models that have been rescaled to the low energy data). Information about the observable quantities for the final six events can be seen in Table III. The final $N_{\text {ch }}$ distribution is shown in Figure 4. The total number of events predicted for the signal and background can be compared to the observed data in Table VI $\left(N_{\text {ch }}<100\right)$ and in Table VII $\left(N_{\mathrm{ch}} \geq 100\right)$. With uncertainties included, the upper limit on a diffuse $\Phi \propto \mathrm{E}^{-2}$ flux of muon neutrinos at Earth (90\% confidence level) with the AMANDA-II detector for 2000 2003 is $7.4 \times 10^{-8} \mathrm{GeV} \mathrm{cm}^{-2} \mathrm{~s}^{-1} \mathrm{sr}^{-1}$ for $16 \mathrm{TeV}$ to $2.5 \mathrm{PeV}$. The results are compared to other neutrino limits in Figure 8 . 


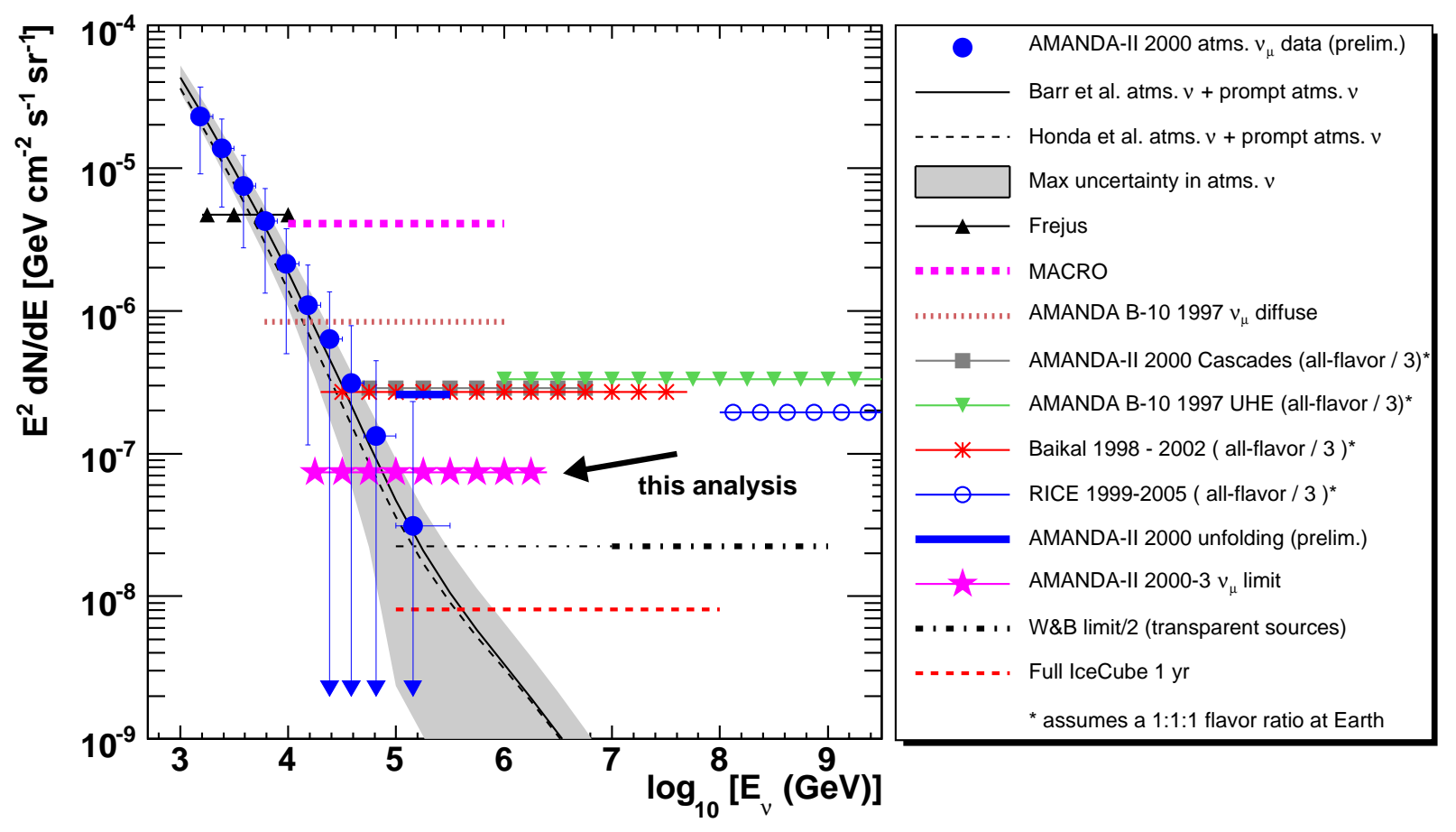

FIG. 8: The upper limits on the $\nu_{\mu}$ flux from sources with an $E^{-2}$ energy spectrum are shown for single and all-flavor analyses. All-flavor upper limits have been divided by three, assuming that the neutrino flavor ratio is 1:1:1 at Earth. The Fréjus [12], MACRO [13], and AMANDA-B10 [10] upper limits on the $\nu_{\mu}$ flux are shown, as well as the unfolded atmospheric spectrum from 2000 AMANDA-II data [42]. The AMANDA-II all-flavor limit from 2000 [11], the AMANDA-B10 UHE limit [43], the Baikal five year limit [14] and the RICE six year limit [44] have all been adjusted for the single flavor plot. The $\Phi \propto \mathrm{E}^{-2}$ limit from this analysis is a factor of four above the WaxmanBahcall upper bound. Although not shown, this analysis excludes the $\Phi \propto \mathrm{E}^{-2}$ predictions made by Nellen, Mannheim and Biermann [4] and Becker, Biermann and Rhode [5] and constrains the MPR upper bound for optically thick pion photoproduction sources [6]. The IceCube sensitivity for a full detector was estimated with AMANDA software [45].

\section{B. Results for Other Energy Spectra}

Other signal models were also tested with this data set. Due to their different energy spectra, the $N_{\text {ch }}$ requirement was reoptimized by minimizing the MRF with each signal model. For signal models with softer spectra than $\Phi \propto \mathrm{E}^{-2}$, a lower $N_{\text {ch }}$ requirement was 


\begin{tabular}{|r|r|r|r|r|r|r|r|}
\hline Event & $\mathbf{1}$ & $\mathbf{2}$ & $\mathbf{3}$ & $\mathbf{4}$ & $\mathbf{5}$ & $\mathbf{6}$ & $\begin{array}{r}\text { required } \\
\text { value }\end{array}$ \\
\hline Year & 2001 & 2001 & 2001 & 2001 & 2002 & 2003 & \\
\hline$N_{\mathrm{ch}}$ & 102 & 106 & 157 & 116 & 100 & 111 & $\geq 100$ \\
\hline Day of Year & 118 & 186 & 210 & 274 & 226 & 182 & \\
\hline Track Length [m] & 206.7 & 221.8 & 197.7 & 178.2 & 180.4 & 207.6 & $>170$ \\
\hline Number of & & & & & & & \\
Direct Hits & 27 & 32 & 30 & 22 & 29 & 29 & $>13$ \\
\hline Zenith Angle [ ${ }^{\circ}$ & 107.3 & 121.6 & 106.1 & 101.8 & 123.8 & 113.3 & $>100$ \\
\hline Median Resolution [ [ & 2.4 & 1.4 & 1.8 & 3.0 & 1.6 & 2.8 & $<4.0$ \\
\hline
\end{tabular}

TABLE III: Observable and reconstructed qualities are shown for the final six events. In addition, events fulfilled requirements based on the reconstructed values of their smoothness $(S)$ and their upgoing vs. downgoing likelihood ratios.

\begin{tabular}{|r|r|r|}
\hline Experiment & $\begin{array}{r}\text { Upper Limit } \\
{\left[\mathrm{GeV} \mathrm{cm}^{-2} \mathrm{~s}^{-1} \mathrm{sr}^{-1}\right]}\end{array}$ & $\begin{array}{l}\text { Energy Range } \\
\log _{10}\left[\mathrm{E}_{\nu}(\mathrm{GeV})\right]\end{array}$ \\
\hline Muon neutrinos only \\
\hline Fréjus [12] & $5.0 \times 10^{-6}$ & $\sim 3.4$ \\
\hline MACRO [13] & $4.1 \pm 0.4 \times 10^{-6}$ & $4.0-6.0$ \\
\hline AMANDA-B10 [10] & $8.4 \times 10^{-7}$ & $3.8-6.0$ \\
\hline AMANDA-II (this analysis) & $7.4 \times 10^{-8}$ & $4.2-6.4$ \\
\hline All neutrino flavors \\
\hline Baikal [14] & $8.1 \times 10^{-7}$ & $4.3-7.7$ \\
\hline AMANDA-B10 [43] & $0.99 \times 10^{-6}$ & $6.0-9.5$ \\
\hline AMANDA-II [11] & $8.6 \times 10^{-7}$ & $4.7-6.7$ \\
\hline
\end{tabular}

TABLE IV: Upper limits for the diffuse flux of extraterrestrial neutrinos as reported by a number of experiments. The first four analyses only constrain the flux of $\nu_{\mu}+\bar{\nu}_{\mu}$, while the last three constrain the total neutrino flux, $\left(\nu_{e}+\bar{\nu}_{e}+\nu_{\mu}+\bar{\nu}_{\mu}+\nu_{\tau}+\bar{\nu}_{\tau}\right)$.

optimal, $N_{\mathrm{ch}} \geq 71$. Four prompt neutrino models [30, 31, 32, 34] and one astrophysical neutrino model [9] were tested under these conditions. One astrophysical model was optimized at $N_{\mathrm{ch}} \geq 86$ [6]. Two astrophysical neutrino models with harder spectra than $\Phi \propto \mathrm{E}^{-2}$ were tested with a higher energy requirement, $N_{\mathrm{ch}} \geq 139[\underline{6}, \mathbf{7},[$ ]] .

Results of these searches are summarized in Table $\mathrm{V}$. The normalization of the overall number of low energy atmospheric neutrinos to data was performed over the region $50<N_{\text {ch }}<100$ for the harder spectra $\left(N_{\text {ch }} \geq 139\right)$, and over $50<N_{\text {ch }}<71$ and $50<N_{\text {ch }}<86$ for the softer spectra.

When the data from the $N_{\mathrm{ch}} \geq 139$ region were examined, there was good agreement 
with the expected atmospheric neutrino background (1 event observed on a backround of 1.55). For $N_{\mathrm{ch}} \geq 86,14$ events were observed while an average of 12.9 background events were predicted. However, 37 events were observed while only 27.4 events were expected for $N_{\mathrm{ch}} \geq 71$, leading to a two-sided confidence interval. Since the chance probability of observing 37 or more events on this background is 4\%, we do not exclude the backgroundonly null hypothesis. The $90 \%$ confidence interval for $\mu$ is shown for each model in Table $\mathrm{V}$ and upper limits are calculated based on the upper bound of each confidence interval. If the MRF is greater than 1, then the model is not ruled out based on observations from this four-year data set. Since more events were observed in the data than were predicted by the background simulation for $N_{\mathrm{ch}} \geq 71$, the upper limit on those five models is roughly a factor of three worse than the sensitivity.

\section{Astrophysical Neutrinos}

The first astrophysical neutrino model tested with the $N_{\mathrm{ch}} \geq 139$ requirement was initially proposed by Stecker, Done, Salamon and Sommers [7]. The flux tested in this analysis includes the revision in the erratum of their original paper [7] and the factor of 20 reduction by Stecker in 2005 [8]. The model predicts a flux $\left(\Phi_{\text {SDSS }}\right)$ of high energy neutrinos from the cores of AGNs, especially Seyfert galaxies. Based on the present data, the upper limit on this flux is $1.6 \cdot \Phi_{\mathrm{SDSS}}$. The best previous limit on this model was established by the Baikal experiment, with an upper limit of $2.5 \cdot \Phi_{\text {SDSS }}[14]$.

Mannheim, Protheroe and Rachen (MPR) [6] computed an upper bound for neutrinos from generic optically thin pion photoproduction sources $\left(\tau_{n \gamma}<1\right)$, as well as an upper bound for neutrinos from AGN jets. (In addition, they calculated an upper bound for generic optically thick $\left(\tau_{n \gamma} \gg 1\right)$ pion photoproduction sources assuming a $\Phi \propto \mathrm{E}^{-2}$ spectrum, but this is constrained by the results discussed in the previous section.) The upper bounds do not necessarily represent physical neutrino energy spectra, but were constructed by taking the envelope of the ensemble of predictions for smaller energy ranges. Each flux prediction within the ensemble was normalized to the observed cosmic ray proton spectrum.

Nonetheless, the shapes of these two upper bounds were tested as if they were models. However, one should be careful not to misinterpret the results. A limit on a model implies a change in the normalization of the entire model. A limit on an upper bound only implies 
a change in normalization of the bound in the energy region where the detector energy response to that spectral shape peaks.

The MPR AGN jet upper bound was tested with the $N_{\mathrm{ch}} \geq 139$ requirement. The upper

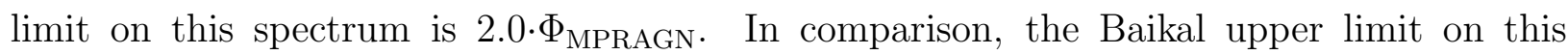
spectrum is $4.0 \cdot \Phi_{\text {MPRAGN }}$.

The MPR upper bound for optically thin sources was tested with a $N_{\mathrm{ch}} \geq 86$ requirement. The limit on this spectrum and normalization is $0.22 \cdot \Phi_{\mathrm{MPR} \tau<1}$.

The remaining neutrino searches were conducted with the lower $N_{\mathrm{ch}}$ requirement, $N_{\text {ch }} \geq 71$. A signal hypothesis involving neutrinos from starburst galaxies [9] was tested. Loeb and Waxman assumed that protons in starburst galaxies with energy less than $3 \mathrm{PeV}$ convert almost all of their energy into pions. Their work predicts a range that should encompass the true neutrino spectrum, but the model tested here uses the most probable spectrum from the paper, $\Phi \propto \mathrm{E}^{-2.15}$. This analysis assumed the flux was valid for energies ranging from $10^{3}$ to $10^{7} \mathrm{GeV}$. The upper limit on this spectral shape and normalization is $21.1 \cdot \Phi_{\text {starburst }}$.

These astrophysical neutrino models and their observed upper limits based on this data set are shown in Figure 9. Neutrino oscillations are taken into account for all models where this factor was not already applied.

\section{Prompt Neutrinos}

Since prompt neutrinos have a harder (less steep) spectrum than the conventional atmospheric neutrinos, it is possible to search for a prompt neutrino flux by separating the two event classes in energy. The final $N_{\text {ch }}$ requirement was reoptimized yielding $N_{\mathrm{ch}} \geq 71$ and the normalization factor was determined based on the interval $\left(50 \leq N_{\mathrm{ch}}<71\right)$.

In the astrophysical neutrino searches described thus far, the range of atmospheric neutrinos predicted in the final sample included an uncertainty due to the unknown prompt neutrino flux. For the search for prompt neutrinos, this uncertainty in the total atmospheric neutrino flux was changed so that only conventional atmospheric neutrino uncertainties were included. Since the atmospheric neutrino simulation was still normalized to the low energy data, the overall effect in the atmospheric background prediction for the final sample was small. 


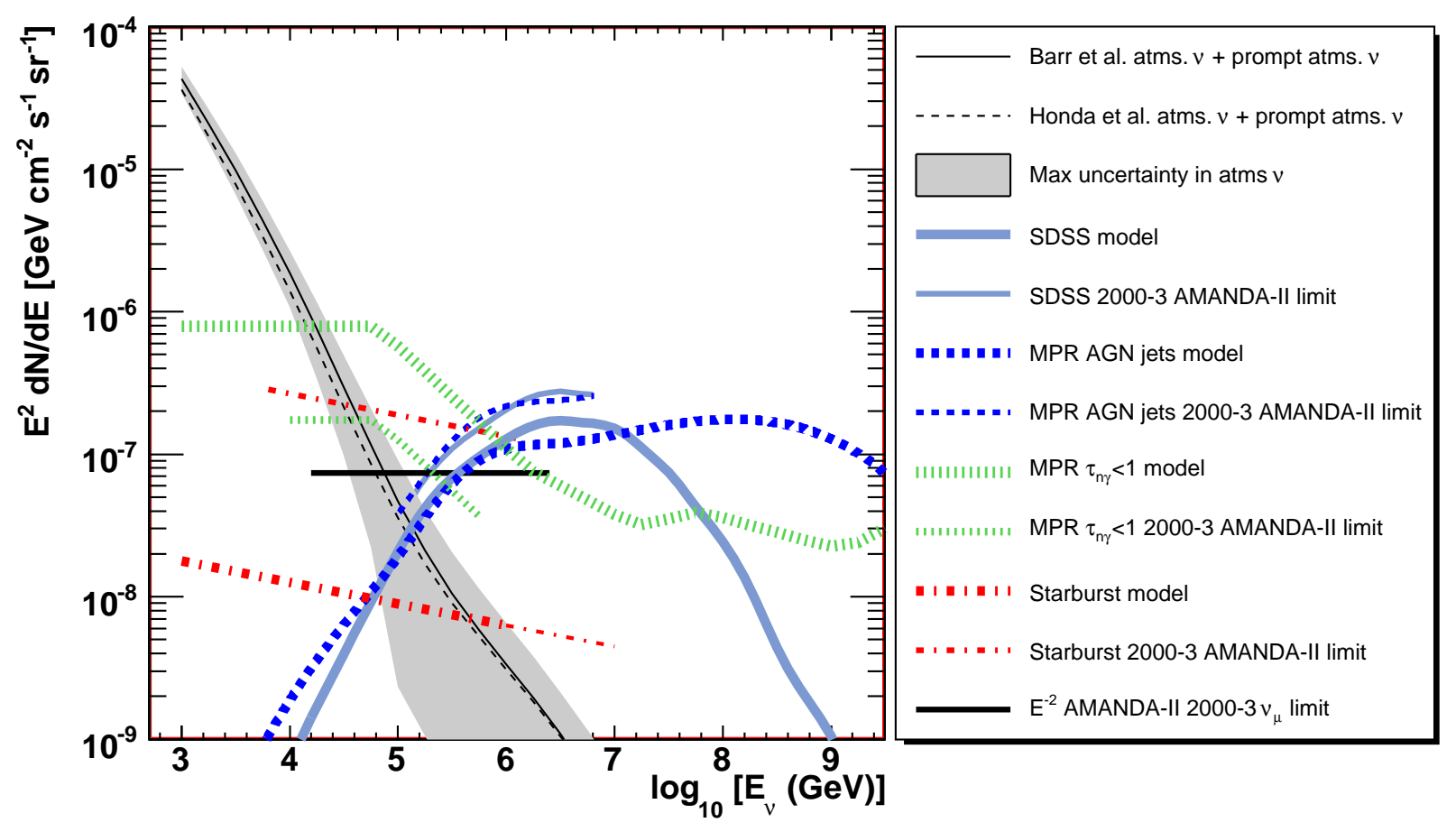

FIG. 9: Astrophysical neutrino models and upper limits established with this analysis. The Barr et al. and Honda et al. atmospheric neutrino models are shown as thin lines with maximum uncertainties assumed by this analysis represented by the band. Other models that were tested included the SDSS AGN core model [7, 8], the MPR upper bounds for AGN jets and optically thin sources [6], and a starburst galaxy model [9].

Martin et al. predict prompt lepton fluxes based on the GBW model for deep inelastic scattering. This model includes gluon saturation effects [30] which lower the predicted charm production cross sections. The predicted flux is lower than the sensitivity of this data set. The upper limit on this model is $60.3 \cdot \Phi_{\text {MartinGBW }}$.

The Naumov RQPM [31, 32] model of prompt atmospheric neutrinos incorporates data from primary cosmic ray and hadronic interaction experiments. This non-perturbative model includes intrinsic charm [33]. The upper limit on this model is $5.2 \cdot \Phi_{\text {NaumovRQPM }}$.

Prompt neutrinos based on the models of Zas, Halzen and Vazquez were also simulated [34]. A parameterization was established to describe the energy dependence of the charm cross section. For the Charm $\mathrm{C}$ model, the charm cross section was fitted to experimental data. In the Charm D model, the cross section was parameterized by Volkova [41]. Due 


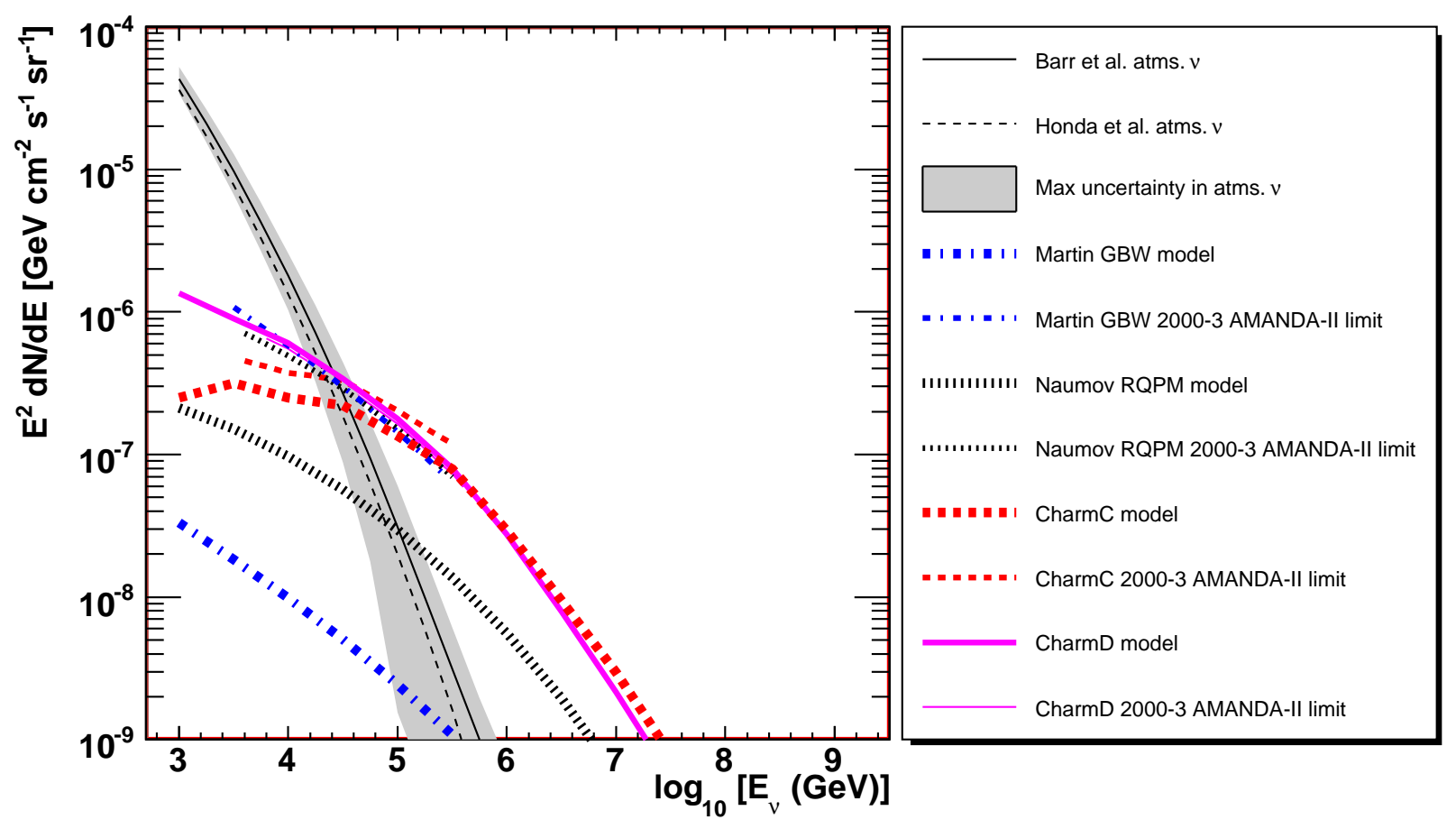

FIG. 10: Prompt neutrino models and upper limits based on this analysis. The Barr et al. and Honda et al. atmospheric neutrino predictions are shown for reference. Two charm models [34] were tested, along with the Naumov RQPM [31, 32] and Martin GBW [30] models.

to the upward fluctuation in the number of events in the $N_{\mathrm{ch}} \geq 71$ region, the upper limit for Charm $\mathrm{C}$ is $1.5 \cdot \Phi_{\mathrm{CharmC}}$. The upper limit on the Charm D model is $0.95 \cdot \Phi_{\mathrm{CharmD}}$. The MRF is less than 1.0, hence the Charm D model is disfavored at the $90 \%$ confidence level.

The prompt neutrino models are shown in Figure 10, along with the upper limits based on these data.

\section{CONCLUSIONS}

The experimental data were consistent with the predicted range of atmospheric neutrino background. Six high energy events were observed in the final data set, while the average predicted background was 7.0 events. There is no indication of an astrophysical signal. At a $90 \%$ confidence level, the diffuse flux of extraterrestrial muon neutrinos with an $\mathrm{E}^{-2}$ spectrum is not larger than $7.4 \times 10^{-8} \mathrm{GeV} \mathrm{cm}^{-2} \mathrm{~s}^{-1} \mathrm{sr}^{-1}$ for $16 \mathrm{TeV}-2.5 \mathrm{PeV}$.

This analysis also provides upper limits on four astrophysical neutrino models and four 
Astrophysical $\nu$

\begin{tabular}{|r|r|r|r|}
\hline & $\Phi \propto E^{-2}$ & SDSS $[7,8]$ & MPR AGN jets $[6]$ \\
\hline$N_{\mathrm{ch}}$ & 100 & 139 & 139 \\
\hline$n_{\mathrm{b}}$ & 7.0 & 1.55 & 1.55 \\
\hline$n_{\mathrm{s}}$ & 66.7 & 1.74 & 1.42 \\
\hline$\mu_{\text {median }}\left(n_{\mathrm{b}}\right)$ & 6.36 & 2.86 & 2.86 \\
\hline sensitivity & & & \\
$\mu_{\text {median }}\left(n_{\mathrm{b}}\right) / n_{\mathrm{s}} \times \Phi$ & $0.095 \times \Phi_{\mathrm{E}^{-2}}$ & $1.6 \times \Phi_{\mathrm{SDSS}}$ & $2.0 \times \Phi_{\mathrm{MPRAGN}}$ \\
\hline$n_{\mathrm{Obs}}$ & 6 & 1 & 1 \\
\hline$\mu_{90 \% \mathrm{C} . \mathrm{I}}$ & $(0,4.95)$ & $(0,2.86)$ & $(0,2.86)$ \\
\hline $\mathrm{upper} \operatorname{limit}_{\mathrm{S}}$ & & & \\
$\mu / n_{\mathrm{s}} \times \Phi$ & $0.074 \times \Phi_{\mathrm{E}^{-2}}$ & $1.6 \times \Phi_{\mathrm{SDSS}}$ & $2.0 \times \Phi_{\mathrm{MPRAGN}}$ \\
\hline$\left(\log _{10} E_{\min }, \log _{10} E_{\max }\right)$ & $(4.2,6.4)$ & $(5.1,6.8)$ & $(5.0,6.9)$ \\
\hline
\end{tabular}

\begin{tabular}{|r|r|r|}
\hline & MPR $\tau_{n \gamma}<1[6]$ & Starburst [9] \\
\hline$N_{\mathrm{ch}}$ & 86 & 71 \\
\hline$n_{\mathrm{b}}$ & 12.9 & 29.1 \\
\hline$n_{\mathrm{s}}$ & 42.7 & 1.05 \\
\hline$\mu_{\text {median }}\left(n_{\mathrm{b}}\right)$ & 8.48 & 8.24 \\
\hline sensitivity & & \\
$\mu_{\text {median }}\left(n_{\mathrm{b}}\right) / n_{\mathrm{s}} \times \Phi$ & $0.2 \times \Phi_{\mathrm{MPR} \tau<1}$ & $7.8 \times \Phi_{\text {Starburst }}$ \\
\hline$n_{\mathrm{Obs}}$ & 14 & 37 \\
\hline$\mu_{90 \% \mathrm{C} . \mathrm{I}}$ & $(0,9.49)$ & $(0,22.13)$ \\
\hline $\mathrm{upper} \operatorname{limit}$ & & \\
$\mu / n_{\mathrm{s}} \times \Phi$ & $0.22 \times \Phi_{\mathrm{MPR} \tau<1}$ & $21.1 \times \Phi_{\text {Starburst }}$ \\
\hline$\left(\log _{10} E_{\min }, \log _{10} E_{\max }\right)$ & $(4.0,5.8)$ & $(3.8,6.1)$ \\
\hline
\end{tabular}

\section{Prompt $\nu$}

\begin{tabular}{|r|r|r|r|r|}
\hline & $\begin{array}{r}\text { Martin } \\
\text { GBW [30] }\end{array}$ & $\begin{array}{r}\text { Naumov } \\
\text { RQP [31, 32] }\end{array}$ & CharmC [34] & CharmD [34] \\
\hline$N_{\mathrm{ch}}$ & 71 & 71 & 71 & 71 \\
\hline$n_{\mathrm{b}}$ & 27.4 & 27.4 & 27.4 & 27.4 \\
\hline$n_{\mathrm{s}}$ & 0.41 & 4.74 & 16.05 & 26.15 \\
\hline$\mu_{\text {median }}\left(n_{\mathrm{b}}\right)$ & 8.75 & 8.75 & 8.75 & 8.75 \\
\hline sensitivity & & & & \\
\hline$\mu_{\text {median }}\left(n_{\mathrm{b}}\right) / n_{\mathrm{s}} \times \Phi$ & $21.3 \times \Phi_{\mathrm{MGBW}}$ & $1.8 \times \Phi_{\mathrm{NRQPM}}$ & $0.55 \times \Phi_{\mathrm{CharmC}}$ & $0.33 \times \Phi_{\mathrm{CharmD}}$ \\
\hline$n_{\mathrm{obs}}$ & 37 & 37 & 37 & 37 \\
\hline$\mu_{90 \% \text { C.I. }}$ & $(1.29,24.72)$ & $(1.29,24.72)$ & $(1.29,24.72)$ & $(1.29,24.72)$ \\
\hline $\mathrm{upper} \operatorname{limit}_{\mathrm{m}}$ & & & & \\
$\mu / n_{\mathrm{s}} \times \Phi$ & $60.3 \times \Phi_{\mathrm{MGBW}}$ & $5.2 \times \Phi_{\mathrm{NRQPM}}$ & $1.5 \times \Phi_{\mathrm{CharmC}}$ & $0.95 \times \Phi_{\mathrm{CharmD}}$ \\
\hline$\left(\log _{10} E_{\text {min }}, \log _{10} E_{\text {max }}\right)$ & $(3.5,5.5)$ & $(3.6,5.6)$ & $(3.8,5.7)$ & $(3.6,5.6)$ \\
\hline
\end{tabular}

TABLE V: Several flux shapes were tested with this data set. $N_{\mathrm{ch}}$ is the minimum number of OMs that had to be hit for an event to appear in the final data set. The predicted number of events for background, $n_{\mathrm{b}}$, and signal, $n_{\mathrm{s}}$, were determined by the simulation. The median event upper limit is $\mu_{\text {median }}\left(n_{\mathrm{b}}\right)$. The sensitivity is the model flux multiplied by the median event upper limit and divided by the number of signal predicted. The number of events observed in the four year data sample is $n_{\text {obs }}$. The upper limit is calculated from the maximum value of the $90 \%$ confidence interval for the event upper limit, $\mu$. The upper limit is the test flux multiplied by $\mu / n_{\mathrm{s}}$. All values quoted here incorporate systematic uncertainties. 
prompt neutrino models. For the hardest signal spectra, the results are consistent with background. The softer spectra were tested with lower $N_{\mathrm{ch}}$ requirements and despite the observation leading to a two-sided $90 \%$ confidence interval, the level of excess is not significant enough to claim a detection.

Before requiring events to fulfill $N_{\mathrm{ch}} \geq 100$, the observed events were compared to the atmospheric neutrino simulation with systematic uncertainties included. The observed low energy data were used to normalize the atmospheric neutrino simulation, hence narrowing the range of atmospheric neutrinos predicted by the different models for the final high energy sample. Systematic effects of the event selection procedure were studied in the inverted analysis using atmospheric muons. A consistency was established between the observed downgoing atmospheric muon flux and the upgoing atmospheric neutrino flux using the inverted analysis.

This result is the best upper limit on the diffuse flux of muon neutrinos to date. The upper limit is an order of magnitude lower than the previous AMANDA result by performing a multi-year analysis [10] and by using a larger detector, AMANDA-II instead of AMANDAB10. For a $\Phi \propto \mathrm{E}^{-2}$ spectral shape, this analysis provides an upper limit that is a factor of three better than the Baikal muon neutrino upper limit (muon neutrino upper limit = all-flavor limit/3 assuming a 1:1:1 flavor ratio).

This analysis set upper limits on four prompt atmospheric neutrino predictions, while one of these models is disfavored at a 90\% confidence level. Other spectral shapes were tested for astrophysical neutrinos. No models were excluded, however constraints were placed on the existing predictions. The shapes of the MPR upper bounds were tested in the energy region where the detector response peaks. For the benchmark $\Phi \propto \mathrm{E}^{-2}$ spectral shape, the current limit is a factor of 4 above the Waxman-Bahcall upper bound.

AMANDA-II has now been integrated into IceCube. The sensitivity of the IceCube detector will continue to improve as the detector grows to its final volume, $1 \mathrm{~km}^{3}$. Based on estimations with AMANDA software, the full IceCube detector will have a sensitivity that is a factor of 10 better than this analysis after one year of operation [45]. 


\begin{tabular}{|c|c|c|c|c|c|c|}
\hline \multicolumn{7}{|c|}{ Upgoing $\mathbf{0}<N_{\mathrm{ch}}<\mathbf{1 0 0}$} \\
\hline & L0 & L1 & L2 & L3 & L4 & L5 \\
\hline signal & & 979 & 664 & 486 & 437 & 141 \\
\hline coinc. $\mu$ & & 60800 & 5750 & 530 & 248 & 0 \\
\hline $\begin{array}{r}\text { misreconstructed } \\
\text { CORSIKA } \\
\text { atms. } \mu\end{array}$ & & 1340000 & 94900 & 1760 & 208 & 0 \\
\hline $\begin{array}{r}\text { Barr et al. } \\
\text { atms. } \nu\end{array}$ & & 9090 & 6590 & 4470 & 3890 & 534 \\
\hline $\begin{array}{r}\text { Honda et al. } \\
\text { atms. } \nu\end{array}$ & & 7290 & 5300 & 3600 & 3130 & 420 \\
\hline $\begin{array}{r}\text { Martin GBW } \\
\text { prompt atms. } \nu\end{array}$ & & 8.2 & 8.2 & 6.4 & 5.7 & 1.2 \\
\hline $\begin{array}{l}\text { Naumov RQPM } \\
\text { prompt atms. } \nu\end{array}$ & & 71.6 & 71.6 & 56.7 & 50.7 & 11.5 \\
\hline Data & & 3956810 & 294947 & 10841 & 4088 & 459 \\
\hline \multicolumn{7}{|c|}{ Downgoing $0<N_{\mathrm{ch}}<100$} \\
\hline $\begin{array}{r}\text { CORSIKA } \\
\text { atms. } \mu\left(\times 10^{7}\right) \\
\end{array}$ & 386 & & 288 & 212 & 195 & 24 \\
\hline data $\left(\times 10^{7}\right)$ & 432 & & 323 & 255 & 229 & 30 \\
\hline
\end{tabular}

TABLE VI: The number of low energy events $\left(0<N_{\mathrm{ch}}<100\right)$ at a given quality level for the different types of simulation and experimental data.

\section{Acknowledgments}

We acknowledge the support from the following agencies: National Science FoundationOffice of Polar Program, National Science Foundation-Physics Division, University of Wisconsin Alumni Research Foundation, Department of Energy, and National Energy Research Scientific Computing Center (supported by the Office of Energy Research of the Department of Energy), the NSF-supported TeraGrid system at the San Diego Supercomputer Center (SDSC), and the National Center for Supercomputing Applications (NCSA); Swedish Research Council, Swedish Polar Research Secretariat, and Knut and Alice Wallenberg Foundation, Sweden; German Ministry for Education and Research, Deutsche Forschungsgemeinschaft (DFG), Germany; Fund for Scientific Research (FNRS-FWO), Flanders Institute to encourage scientific and technological research in industry (IWT), Belgian Federal Office for Scientific, Technical and Cultural affairs (OSTC); the Netherlands Organisation for Scientific Research (NWO); M. Ribordy acknowledges the support of the SNF (Switzerland); A. 


\begin{tabular}{|r|r|r|r|r|r|r|}
\hline \multicolumn{6}{|c|}{ Upgoing $N_{\text {ch }} \geq 100$} \\
\hline signal & L0 & L1 & L2 & L3 & L4 & L5 \\
\hline coinc. $\mu$ & & 54.2 & 4.3 & 2.8 & 2.8 & 0 \\
\hline $\begin{array}{r}\text { misreconstructed } \\
\text { CORSIKA } \\
\text { atms. } \mu\end{array}$ & & 862 & 35.4 & 0 & 0 & 0 \\
\hline $\begin{array}{r}\text { Barr } \text { et al. } \\
\text { atms. } \nu\end{array}$ & & 36.0 & 27.6 & 19.3 & 18.9 & 9.1 \\
\hline $\begin{array}{r}\text { Honda } \text { et al. } \\
\text { atms. } \nu\end{array}$ & & 25.2 & 19.3 & 13.5 & 13.2 & 6.4 \\
\hline $\begin{array}{r}\text { Martin GBW } \\
\text { prompt atms. } \nu\end{array}$ & 0.42 & 0.42 & 0.36 & 0.36 & 0.19 \\
\hline $\begin{array}{r}\text { Naumov RQPM } \\
\text { prompt atms. } \nu\end{array}$ & & & & & & \\
\hline Data & & 11456 & 1347 & 96 & 45 & 6 \\
\hline Downgoing $N_{\text {ch }} \geq 100$ & 4.2 & 4.2 & 2.2 \\
\hline $\begin{array}{r}\text { CORSIKA } \\
\mu\end{array}$ & & & & & \\
\hline atms. $\left.\mu 10^{7}\right)$ & 7.31 & & 6.53 & 6.05 & 6.01 & 5.09 \\
\hline data $\left(\times 10^{7}\right)$ & 9.75 & & 8.59 & 8.07 & 8.03 & 6.60 \\
\hline \multicolumn{7}{|c|}{ L5 level of final analysis }
\end{tabular}

TABLE VII: The number of high energy events $\left(N_{\mathrm{ch}} \geq 100\right)$ at a given quality level for the different types of simulation and experimental data.

Kappes and J. D. Zornoza acknowledge support by the EU Marie Curie OIF Program. 


\section{Appendix A: Event Selection Techniques}

Event selection techniques were applied to find the best reconstructed upgoing tracks. The event requirements were tightened through a series of values, becoming more restrictive at each of the five different levels. As seen in Figure 11, requiring a minimum value of the track length, for instance, can be a powerful method of rejecting misreconstructed downgoing backgrounds. The event selection requirements for $L_{\text {dir }}, N_{\text {dir }}$, smoothness, median resolution and likelihood ratio were established to remove many orders of magnitude more misreconstructed background than upgoing atmospheric neutrinos or signal neutrinos. Events which did not meet an optimized minimum or maximum value of each parameter were removed.

The strength of these quality requirements was adjusted at each level. The requirement is defined for each parameter in Table VIII. The plots in Figure 3 show the zenith angle distribution of all events fulfilling the zenith angle $>80^{\circ}$ and event observable requirements at the chosen level. After the zenith angle criteria was fulfilled at Level 1, the data mostly contains misreconstructed atmospheric muons (top right, Figure 3). As the quality parameters become more restrictive, the data begins to follow the atmospheric neutrino simulation in the upgoing direction and the atmospheric muon simulation in the downgoing direction. At Level 5, the event quality requirements were strong enough to have removed all of the misreconstructed downgoing atmospheric muon events that were simulated. However, to be sure that the final data set only included atmospheric and astrophysical neutrinos and no misreconstructed downgoing events, an additional zenith angle requirement was imposed. All events were kept if they were reconstructed between $100^{\circ}$ and $180^{\circ}$. The analysis continued with the data sample shown at Level 5. 


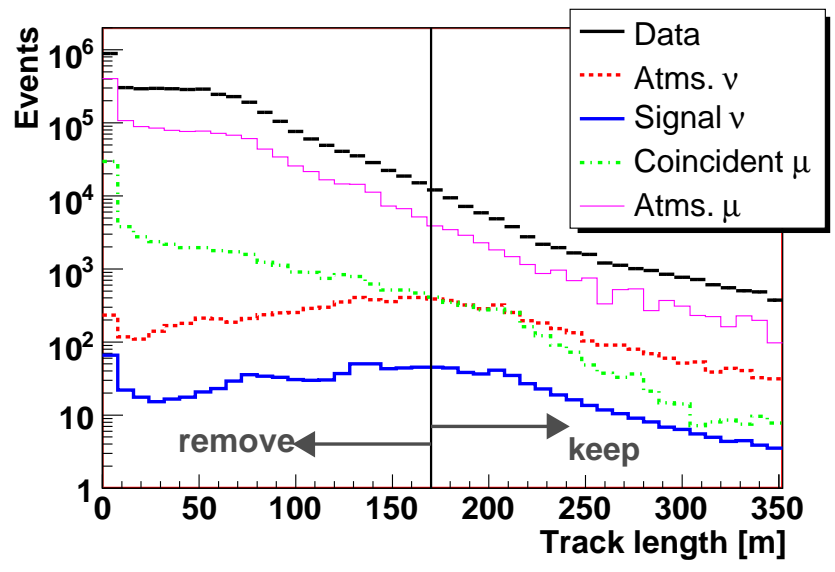

FIG. 11: The reconstructed track length within the detector is shown. In order to identify muon neutrino tracks, events were required to have long tracks of at least 170 meters. This removed a large fraction of the atmospheric muon simulation, but had a smaller effect on the atmospheric neutrino and signal simulations.

\begin{tabular}{|c|c|c|c|c|c|c|}
\hline & L0 & L1 & L2 & L3 & $\overline{\mathrm{L} 4}$ & L5* \\
\hline Zenith Angle $\left[^{\circ}\right]$ & & $>80$ & $>80$ & $>80$ & $>80$ & $>100$ \\
\hline $\begin{array}{l}\text { Number of } \\
\text { Direct Hits }\end{array}$ & & & $>5$ & $>8$ & $>8$ & $>13$ \\
\hline Track Length $[\mathrm{m}]$ & & & $>100$ & $>130$ & $>130$ & $>170$ \\
\hline $\mid$ Smoothness & & & & $<0.30$ & $<0.30$ & $<0.25$ \\
\hline $\begin{array}{c}\text { Median } \\
\text { Resolution }\left[{ }^{\circ}\right]\end{array}$ & & & & & $<4.0$ & $<4.0$ \\
\hline $\begin{array}{c}\text { Likelihood } \\
\text { Ratio }(\Delta L) \\
\text { vs. Zenith }\end{array}$ & & & & & & $\Delta L>-38.2 \cos ($ zenith $)+27.506$ \\
\hline $\begin{array}{c}\text { Number of } \\
\text { Remaining Events }\end{array}$ & $5.2 \times 10^{9}$ & $7.8 \times 10^{6}$ & $1.2 \times 10^{6}$ & $3.5 \times 10^{5}$ & $1.8 \times 10^{5}$ & 465 \\
\hline
\end{tabular}

TABLE VIII: The table summarizes the event quality requirements as a function of quality level. Events only remained in the sample if they fulfilled all of the parameter requirements for a given level. The removal of all horizontal events (zenith $<100$ ) contributed to the large decrease in events from L4 to L5.

\section{Appendix B: Neutrino Effective Area}




\begin{tabular}{|c|c|c|c|c|}
\hline \multirow{2}{*}{$\begin{array}{c}\text { Energy } \\
\log _{10}(\mathrm{E} / \mathrm{GeV})\end{array}$} & \multicolumn{2}{|c|}{$-1<\cos ($ Zenith $)<-.8$} & \multicolumn{2}{|c|}{$-.8<\cos ($ Zenith $)<-.6$} \\
\hline & $\begin{array}{c}\nu_{\mu} \\
{\left[10^{3} \mathrm{~cm}^{2}\right]}\end{array}$ & $\begin{array}{c}\bar{\nu}_{\mu} \\
{\left[10^{3} \mathrm{~cm}^{2}\right]}\end{array}$ & $\begin{array}{c}\nu_{\mu} \\
{\left[10^{3} \mathrm{~cm}^{2}\right]}\end{array}$ & $\begin{array}{c}\bar{\nu}_{\mu} \\
{\left[10^{3} \mathrm{~cm}^{2}\right]}\end{array}$ \\
\hline 3.6 & 0.487 & 0.166 & 0.279 & 0.0673 \\
\hline 3.8 & 1.04 & 1.1 & 0.652 & 0.646 \\
\hline 4 & 3.36 & 2.85 & 1.82 & 1.89 \\
\hline 4.2 & 8.74 & 7.54 & 4.97 & 5.56 \\
\hline 4.4 & 18.8 & 16.2 & 15.3 & 12.4 \\
\hline 4.6 & 29.3 & 30.4 & 34 & 26.9 \\
\hline 4.8 & 44.9 & $\overline{46.4}$ & $\overline{52.7}$ & 58.8 \\
\hline 5 & $\overline{59.6}$ & 65.5 & $\overline{92.6}$ & 88 \\
\hline 5.2 & 75.7 & 69.7 & 128 & 121 \\
\hline 5.4 & 72.6 & 84.4 & 153 & 163 \\
\hline 5.6 & 63.5 & 77.8 & 180 & 179 \\
\hline 5.8 & 63.3 & 66.9 & 183 & 188 \\
\hline 6 & 51.9 & 49.3 & 170 & 177 \\
\hline 6.2 & 36.6 & 39.1 & 145 & 151 \\
\hline 6.4 & 27.8 & 22.6 & 110 & 113 \\
\hline 6.6 & 9.97 & 14.7 & 72.3 & 77 \\
\hline 6.8 & 7.8 & 8.73 & 54.2 & 48.2 \\
\hline 7 & 3.39 & 3.08 & 29.6 & 29.5 \\
\hline 7.2 & 3.12 & 1.44 & 16.5 & 15.2 \\
\hline 7.4 & 0.939 & 0.718 & 7.97 & 9.64 \\
\hline 7.6 & 0.864 & 0.791 & 5.12 & 4.15 \\
\hline 7.8 & 0.492 & 0.521 & 2.59 & 2.08 \\
\hline
\end{tabular}

\begin{tabular}{|c|c|c|c|c|}
\hline \multirow{2}{*}{$\begin{array}{c}\text { Energy } \\
\log _{10}(\mathrm{E} / \mathrm{GeV})\end{array}$} & \multicolumn{2}{|c|}{$-.6<\cos ($ Zenith $)<-.4$} & \multicolumn{2}{|c|}{$-.4<\cos ($ Zenith $)<-.17$} \\
\hline & {$\left[\begin{array}{c}\nu_{\mu} \\
{\left[10^{3} \mathrm{~cm}^{2}\right]}\end{array}\right.$} & $\begin{array}{c}\bar{\nu}_{\mu} \\
{\left[10^{3} \mathrm{~cm}^{2}\right]} \\
\end{array}$ & {$\left[\begin{array}{c}\nu_{\mu} \\
{\left[10^{3} \mathrm{~cm}^{2}\right]}\end{array}\right.$} & $\begin{array}{c}\bar{\nu}_{\mu} \\
{\left[10^{3} \mathrm{~cm}^{2}\right]}\end{array}$ \\
\hline 3.6 & 0.108 & 0.0562 & 0.0752 & 0.0451 \\
\hline 3.8 & 0.282 & 0.163 & 0.178 & 0.0818 \\
\hline 4 & 0.845 & 0.93 & 1.13 & 0.543 \\
\hline 4.2 & 3.73 & 3.39 & 1.98 & 1.66 \\
\hline 4.4 & 9.74 & 8.22 & 7.23 & 6.02 \\
\hline 4.6 & 21.1 & 19.9 & 17.9 & 18.2 \\
\hline 4.8 & 49.7 & 43.3 & 33.2 & 36.9 \\
\hline 5 & 86.2 & 77.5 & 74.2 & 68.3 \\
\hline 5.2 & 118 & 119 & 119 & 113 \\
\hline 5.4 & 179 & 165 & 163 & 167 \\
\hline 5.6 & 232 & 217 & 264 & 230 \\
\hline 5.8 & 243 & 232 & 306 & 310 \\
\hline 6 & 271 & 286 & 377 & 373 \\
\hline 6.2 & 269 & 258 & 418 & 389 \\
\hline 6.4 & 251 & 229 & 441 & 452 \\
\hline 6.6 & 212 & 197 & 437 & 391 \\
\hline 6.8 & 154 & 149 & 417 & 437 \\
\hline 7 & 105 & 114 & 413 & 380 \\
\hline 7.2 & 79.8 & 61.4 & 328 & 327 \\
\hline 7.4 & 46.3 & 32.9 & 285 & 274 \\
\hline 7.6 & 31.8 & 19.4 & 209 & 212 \\
\hline 7.8 & 17.7 & 10.3 & 142 & 146 \\
\hline
\end{tabular}

TABLE IX: Effective area as a function of the energy and zenith angle of the simulation.

\section{Appendix C: Atmospheric Neutrino Flux}

For this analysis, the atmospheric neutrino flux models by Barr et al. and Honda et al. were both considered equally likely options for the background atmospheric neutrino simulation. These two models are among many that use slightly different parameterizations of the all-nucleon cosmic ray flux to derive the atmospheric neutrino flux. 
Effective Area in $\mathbf{c m}^{2}$

\begin{tabular}{|c||c|c||}
\hline \multicolumn{1}{|c||}{ Energy } & \multicolumn{2}{c||}{ All angle } \\
$\log _{10}(\mathrm{E} / \mathrm{GeV})$ & $\nu_{\mu}$ & $\bar{\nu}_{\mu}$ \\
& {$\left[10^{3} \mathrm{~cm}^{2}\right]$} & {$\left[10^{3} \mathrm{~cm}^{2}\right]$} \\
\hline \hline 3.6 & 0.164 & 0.0572 \\
\hline 3.8 & 0.381 & 0.343 \\
\hline 4 & 1.24 & 1.07 \\
\hline 4.2 & 3.33 & 3.15 \\
\hline 4.4 & 8.9 & 7.51 \\
\hline 4.6 & 17.9 & 16.7 \\
\hline 4.8 & 31.8 & 32.6 \\
\hline 5 & 55.6 & 52.7 \\
\hline 5.2 & 78.9 & 75.8 \\
\hline 5.4 & 102 & 103 \\
\hline 5.6 & 136 & 127 \\
\hline 5.8 & 144 & 145 \\
\hline 6 & 161 & 162 \\
\hline 6.2 & 164 & 155 \\
\hline 6.4 & 157 & 155 \\
\hline 6.6 & 139 & 130 \\
\hline 6.8 & 121 & 126 \\
\hline 7 & 112 & 102 \\
\hline 7.2 & 86 & 83.4 \\
\hline 7.4 & 67.8 & 63.6 \\
\hline 7.6 & 51.2 & 49 \\
\hline 7.8 & 35.5 & 34.6 \\
\hline
\end{tabular}

TABLE X: The angle-averaged neutrino effective area as a function of energy.

For this analysis, the Barr et al. flux below $10 \mathrm{GeV}$ was taken from [21]. From $10 \mathrm{GeV}$ to $10 \mathrm{TeV}$, the flux tables from [22], based on the primary spectrum of [23], were used. Above $10 \mathrm{TeV}$, the weight was derived by performing a 2-dimensional fit with a fifth degree polynomial to the $\log _{10} \mathrm{E}$ vs. $\cos ($ zenith) tables of the atmospheric neutrino flux values from lower energies just mentioned. The TARGET version 2.1 [46] hadronic interaction model was used [21].

In an attempt to better fit the AMS [47] and BESS [48, 49] data, Honda et al. changed the power law fit to the proton cosmic ray spectrum from -2.74 to -2.71 above $100 \mathrm{GeV}[20]$. Other parameters in the cosmic ray fit remained similar to the Barr et al. flux mentioned above [36], although the DPMJET-III [50] interaction model was used. The atmospheric neutrino weights from [20] were used up to $10 \mathrm{TeV}$. Above that energy, a 2-dimensional 


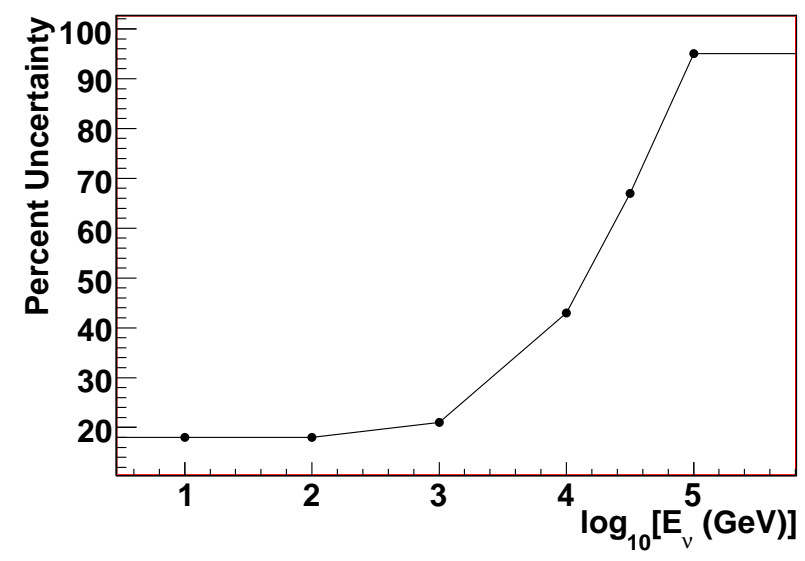

FIG. 12: The estimated uncertainty in the atmospheric neutrino flux as a function of energy. Due to the large uncertainty in the prompt neutrino flux at greater than $10^{4} \mathrm{GeV}$, the total uncertainty rises sharply.

fit of the lower energy values was again used as described above. The result was a lower atmospheric neutrino flux prediction than the Barr et al. flux.

As described in Section VA, uncertainties in hadronic interactions and the cosmic ray and prompt neutrino fluxes at high energy led to large total uncertainties in the atmospheric neutrino flux. The estimated uncertainties are shown in Figure 12,

The atmospheric neutrino simulation was renormalized based on the experimental low energy data. The number of low energy conventional atmospheric neutrinos (second column) is added to the 4.0 prompt neutrinos predicted with the central prompt neutrino model. The total atmospheric background prediction before renormalization is shown in the third column of Table XI. Instead of renormalizing the simulation based on all events with $N_{\mathrm{ch}}<100$, the renormalization was only based on the region $50<N_{\mathrm{ch}}<100$. Because of the difficulty of simulating events near the threshold of the detector, the atmospheric neutrino simulation did not faithfully reproduce the shape of the $N_{\mathrm{ch}}$ distribution for the data at $N_{\mathrm{ch}}$ below 50 . Atmospheric neutrino models were scaled to match the 146 events seen in the experimental data for $50<N_{\mathrm{ch}}<100$. The total number of high energy events predicted to survive the final energy requirement is shown before renormalization in the sixth column and after renormalization in the last column. 


\begin{tabular}{|c|c|c|c|c|c|c|}
\hline Atms. $\nu$ Model & $\begin{array}{c}\text { Conv. } \\
\text { Atms. } \nu \\
50<N_{\mathrm{ch}}<100\end{array}$ & $\begin{array}{c}\text { Conv. } \nu+ \\
\text { prompt } \nu \\
50<N_{\mathrm{ch}}<100\end{array}$ & $\begin{array}{c}\text { Scale Factor } \\
\text { to } 146 \\
\text { Low Energy } \\
\text { Data Events }\end{array}$ & $\begin{array}{c}\text { Conv. } \\
\text { Atms. } \nu \\
N_{\mathrm{ch}} \geq 100\end{array}$ & $\begin{array}{l}\text { Conv. } \nu+ \\
\text { prompt } \nu \\
N_{\mathrm{ch}} \geq 100\end{array}$ & \begin{tabular}{|c} 
Background \\
Predicted in \\
$N_{\mathrm{ch}} \geq 100$ \\
Sample \\
after Scaling
\end{tabular} \\
\hline Barr et al. Max & 249 & 253 & 0.58 & 13.3 & 14.5 & 8.3 \\
\hline Barr et al. & 194 & 198 & 0.74 & 9.1 & 10.3 & 7.6 \\
\hline Barr et al. Min & 138 & 142 & 1.03 & 4.9 & 6.1 & 6.3 \\
\hline Honda et al. Max & 191 & 195 & 0.75 & 9.3 & 10.5 & 7.9 \\
\hline Honda et al. & 149 & 153 & 0.96 & 6.4 & 7.6 & 7.3 \\
\hline Honda et al. Min & 107 & 111 & 1.32 & 3.4 & 4.6 & 6.1 \\
\hline
\end{tabular}

TABLE XI: Number of atmospheric neutrino events predicted by the Monte Carlo. Uncertainty in the high energy cosmic ray flux was incorporated into the maximum and minimum predictions.

[1] E. Waxman and J. Bahcall, Phys. Rev. D 59, 023002 (1998).

[2] J. Bahcall and E. Waxman, Phys. Rev. D 64, 023002 (2001).

[3] E. Waxman, Nucl. Phys. Proc. Suppl. 118, 353 (2003).

[4] L. Nellen, K. Mannheim, and P.L. Biermann, Phys. Rev. D 47, 5270 (1993).

[5] J. Becker, P. Biermann, and W. Rhode, Astropart. Phys. 23, 355 (2005).

[6] K. Mannheim, R.J. Protheroe, and J.P. Rachen, Phys. Rev. D 63, 023003 (2000).

[7] F.W. Stecker, C. Done, M.H. Salamon, and P. Sommers, Phys. Rev. Lett. 66, 2697 (1991); 69, 2738(E) (1992).

[8] F.W. Stecker, Phys. Rev. D 72, 107301 (2005).

[9] A. Loeb and E. Waxman, J. Cosmol. Astropart. Phys. JCAP05 003 (2006).

[10] J. Ahrens et al., Phys. Rev. Lett. 90, 251101 (2003).

[11] M. Ackermann et al., Astropart. Phys. 22, 127 (2004).

[12] W. Rhode et al. (Fréjus Collaboration), Astropart. Phys. 4, 217 (1996).

[13] M. Ambrosio et al. (MACRO Collaboration), Astropart. Phys. 19, 1 (2003).

[14] V. Aynutdinov et al., Astropart. Phys. 25, 140 (2006).

[15] H. Athar, M. Jezabek, and O. Yasuda, Phys. Rev. D 62, 103007 (2000).

[16] A. Achterberg et al., astro-ph 0611063 (2006).

[17] J. Ahrens et al. (AMANDA Collaboration), Nucl. Instr. Meth. A 524, 169 (2004).

[18] D. Heck, J. Knapp, J.N. Capdevielle, G. Schatz, and T. Thouw, Tech. Rep. FZKA 6019, Forschungszentrum Karlsruhe (1998).

[19] G.C. Hill, Astropart. Phys. 6, 215 (1997). 
[20] M. Honda, T. Kajita, K. Kasahara, and S. Midorikawa, Phys. Rev. D 70, 043008 (2004).

[21] G.D. Barr, T.K. Gaisser, P. Lipari, S. Robbins, and T. Stanev, Phys. Rev. D 70, 023006 (2004).

[22] G. Barr, T.K. Gaisser, P. Lipari, S. Robbins, and T. Stanev, http://www-pnp.physics.ox.ac.uk/ barr/fluxfiles/.

[23] T.K. Gaisser, M. Honda, P. Lipari, and T. Stanev, in Proceedings of the 27th International Cosmic Ray Conference, Hamburg, Germany, 5, 1643 (2001).

[24] P. Lipari, Astropart. Phys. 1, 195 (1993).

[25] T. Neunhoffer, Astropart. Phys. 25, 220 (2006).

[26] G.C. Hill and K. Rawlins, Astropart. Phys. 19, 393 (2003).

[27] G.J. Feldman and R.D. Cousins, Phys. Rev. D 57, 3873 (1998).

[28] L. Gerhardt for the IceCube Collaboration, in Proceedings of the 29th International Cosmic Ray Conference, Pune, India, 5, 111 (2005), astro-ph 0509330.

[29] M. Ackermann et al., J. Geophys. Res. 111, D13203 (2006).

[30] A.D. Martin, M.G. Ryskin, and A.M. Stasto, Acta Phys. Polon. B34, 3273 (2003).

[31] G. Fiorentini, A. Naumov, and F.L. Villante, Phys. Lett. B 510, 173 (2001).

[32] E.V. Bugaev et al., Il Nuovo Cimento 12C, No. 1, 41 (1989).

[33] C.G.S. Costa, Astropart. Phys. 16, 193 (2001).

[34] E. Zas, F. Halzen, and R.A. Vázquez, Astropart. Phys. 1, 297 (1993).

[35] T.K. Gaisser and M. Honda, Annu. Rev. Nucl. Part. Sci. 52, 153 (2002).

[36] T.K. Gaisser, in Proceedings of Nobel Symposium 129 "Neutrino Physics", astro-ph 0502380 (2005).

[37] D. Chirkin, hep-ph 0407078 (2004).

[38] R.D. Cousins and V.L. Highland, Nucl. Instrum. Methods Phys. Res. A 320, 331 (1992).

[39] J. Conrad, O. Botner, A. Hallgren, and C. Pérez de los Heros, Phys. Rev. D 67, 012002 (2003).

[40] G.C. Hill, Phys. Rev. D 67, 118101 (2003).

[41] L.V. Volkova et al., Il Nuovo Cimento C10, 465 (1987).

[42] K. Münich for the IceCube Collaboration, in Proceedings of the 29th International Cosmic Ray Conference, Pune, India, 5, 17 (2005), astro-ph 0509330.

[43] M. Ackermann et al., Astropart. Phys. 22, 339 (2005).

[44] I. Kravchenko et al., Phys. Rev. D 73, 082002 (2006). The value of the upper limit is taken 
from Figure 19. S. Hussain (private communication).

[45] J. Ahrens et al., Astropart. Phys. 20, 507 (2004).

[46] R. Engel et al., in Proceedings of the 27th International Cosmic Ray Conference, Hamburg, Germany, 1381 (2001).

[47] J. Alcaraz et al. (AMS Collaboration), Phys. Lett. B 490, 27 (2000).

[48] T. Sanuki et al., Astrophys. J. 545, 1135 (2000).

[49] S. Haino et al., Phys. Lett. B 594, 35 (2004).

[50] S. Roesler, R. Engel, and J. Ranft, in Proceedings of the 27th International Cosmic Ray Conference, Hamburg, Germany, 1, 439 (2001); Phys. Rev. D 57, 2889 (1998). 
The following pages are an erratum to the original document. 


\section{Erratum: Multi-year search for a diffuse flux of muon neutrinos with AMANDA-II}

A search for $\mathrm{TeV}$ - PeV muon neutrinos with AMANDA-II data collected between 2000 and 2003 established an upper limit of $\mathrm{E}^{2} \Phi_{90 \% \text { C.L. }}<7.4 \times 10^{-8} \mathrm{GeV} \mathrm{cm}^{-2} \mathrm{~s}^{-1} \mathrm{sr}^{-1}$ on the diffuse flux of extraterrestrial muon neutrinos with a $\Phi \propto \mathrm{E}^{-2}$ spectrum between $16 \mathrm{TeV}$ and 2.5 PeV. The upper limit calculation correctly included event simulations and remains as stated. However, the calculation of the detector's efficiency, which is based only on simulations, was incorrectly tabulated in an appendix and shown in a figure. The values were approximately a factor of ten too high, although the exact error varies in each bin. The correction has been applied in the following tables and figure. The effective area is the equivalent area over which the detector would be $100 \%$ efficient for detecting neutrinos. The typical uncertainty on the effective area from simulation statistics is lowest between $10^{5}$ $\mathrm{GeV}$ and $10^{6} \mathrm{GeV}(2 \%)$. The uncertainty increases to $6 \%$ at $10^{4} \mathrm{GeV}$ and $5 \%$ around $10^{7}$ $\mathrm{GeV}$. In the remainder of this document, the number of optical modules (OMs) triggered during an event is referred to as $N_{\text {ch }}$ and $\cos \left(\theta_{\mathrm{t}}\right)$ refers to the cosine of the simulated (true) zenith angle of an event. The term angle-averaged indicates that results are averaged over $\theta_{\mathrm{t}}$ between $100^{\circ}$ and $180^{\circ}$. All other results reported in the paper, including the upper limit, remain unchanged. 


\begin{tabular}{|c|c|c|c|c|}
\hline \multirow{2}{*}{$\begin{array}{c}\text { Energy } \\
\log _{10}(\mathrm{E} / \mathrm{GeV})\end{array}$} & \multicolumn{2}{|c|}{$-\mathbf{1 . 0}<\cos \left(\theta_{t}\right)<-0.8$} & \multicolumn{2}{|c|}{$-0.8<\cos \left(\theta_{t}\right)<-0.6$} \\
\hline & {$\left[\begin{array}{c}\nu_{\mu} \\
{\left[10^{3} \mathrm{~cm}^{2}\right]}\end{array}\right.$} & $\begin{array}{c}\bar{\nu}_{\mu} \\
{\left[10^{3} \mathrm{~cm}^{2}\right]}\end{array}$ & {$\left[\begin{array}{c}\nu_{\mu} \\
{\left[10^{3} \mathrm{~cm}^{2}\right]}\end{array}\right.$} & $\begin{array}{c}\bar{\nu}_{\mu} \\
{\left[10^{3} \mathrm{~cm}^{2}\right]}\end{array}$ \\
\hline 3.6 & 0.046 & 0.017 & 0.024 & 0.0084 \\
\hline 3.8 & 0.094 & 0.1 & 0.052 & 0.049 \\
\hline 4 & 0.32 & 0.29 & 0.19 & 0.18 \\
\hline 4.2 & 0.81 & 0.74 & 0.48 & 0.52 \\
\hline 4.4 & 1.7 & 1.5 & 1.4 & 1.1 \\
\hline 4.6 & 2.6 & 2.7 & 2.9 & 2.5 \\
\hline 4.8 & 4 & 4 & 4.8 & 5.2 \\
\hline 5 & 5.3 & 5.7 & 8.2 & 7.6 \\
\hline 5.2 & 6.5 & 6.2 & 11 & 11 \\
\hline 5.4 & 6.4 & 7.4 & 14 & 14 \\
\hline 5.6 & 5.6 & 6.4 & 16 & 16 \\
\hline 5.8 & 5.2 & 6 & 16 & 16 \\
\hline 6 & 4.3 & 4.3 & 15 & 15 \\
\hline 6.2 & 3.3 & 3.3 & 13 & 13 \\
\hline 6.4 & 2.4 & 2 & 9.4 & 9.7 \\
\hline 6.6 & 0.91 & 1.2 & 6.5 & 6.6 \\
\hline 6.8 & 0.71 & 0.66 & 4.3 & 4.2 \\
\hline 7 & 0.37 & 0.28 & 2.6 & 2.7 \\
\hline 7.2 & 0.26 & 0.15 & 1.5 & 1.5 \\
\hline 7.4 & 0.078 & 0.07 & 0.83 & 0.87 \\
\hline 7.6 & 0.074 & 0.047 & 0.45 & 0.49 \\
\hline 7.8 & 0.02 & 0.055 & 0.26 & 0.19 \\
\hline
\end{tabular}

\begin{tabular}{|c||c|c||c|c||}
\hline \multicolumn{2}{|c|}{ Energy } & $\mathbf{- 0 . 6}<\mathbf{c o s}\left(\theta_{\mathrm{t}}\right)<-\mathbf{0 . 4}$ & $-\mathbf{0 . 4}<\mathbf{c o s}\left(\theta_{\mathrm{t}}\right)<-\mathbf{0 . 1 7}$ \\
$\log _{10}(\mathrm{E} / \mathrm{GeV})$ & $\nu_{\mu}$ & $\bar{\nu}_{\mu}$ & $\nu_{\mu}$ & $\bar{\nu}_{\mu}$ \\
& {$\left[10^{3} \mathrm{~cm}^{2}\right]$} & {$\left[10^{3} \mathrm{~cm}^{2}\right]$} & {$\left[10^{3} \mathrm{~cm}^{2}\right]$} & {$\left[10^{3} \mathrm{~cm}^{2}\right]$} \\
\hline \hline 3.6 & 0.0087 & 0.0043 & 0.0055 & 0.0032 \\
\hline 3.8 & 0.035 & 0.018 & 0.015 & 0.01 \\
\hline 4 & 0.081 & 0.087 & 0.11 & 0.037 \\
\hline 4.2 & 0.35 & 0.31 & 0.16 & 0.14 \\
\hline 4.4 & 0.9 & 0.8 & 0.69 & 0.59 \\
\hline 4.6 & 1.9 & 1.9 & 1.5 & 1.6 \\
\hline 4.8 & 4.4 & 4.1 & 3 & 3.2 \\
\hline 5 & 7.5 & 7.1 & 6.8 & 5.8 \\
\hline 5.2 & 11 & 11 & 11 & 10 \\
\hline 5.4 & 16 & 14 & 15 & 14 \\
\hline 5.6 & 20 & 19 & 23 & 20 \\
\hline 5.8 & 22 & 20 & 26 & 27 \\
\hline 6 & 23 & 24 & 32 & 32 \\
\hline 6.2 & 24 & 23 & 37 & 33 \\
\hline 6.4 & 22 & 20 & 38 & 38 \\
\hline 6.6 & 18 & 17 & 37 & 34 \\
\hline 6.8 & 13 & 13 & 36 & 37 \\
\hline 7 & 9.4 & 9.8 & 34 & 31 \\
\hline 7.2 & 6.9 & 5.9 & 27 & 29 \\
\hline 7.4 & 4 & 3.4 & 23 & 23 \\
\hline 7.6 & 2.7 & 1.7 & 16 & 18 \\
\hline 7.8 & 1.1 & 1.1 & 12 & 13 \\
\hline
\end{tabular}

TABLE XII: Effective area as a function of the energy and zenith angle of the simulation for events in the final sample satisfying $N_{\mathrm{ch}} \geq 100$. 


\begin{tabular}{|c||c|c||}
\hline \multicolumn{1}{|c||}{ Energy } & \multicolumn{2}{|c||}{ Angle-averaged } \\
$\log _{10}(\mathrm{E} / \mathrm{GeV})$ & $\nu_{\mu}$ & $\bar{\nu}_{\mu}$ \\
& {$\left[10^{3} \mathrm{~cm}^{2}\right]$} & {$\left[10^{3} \mathrm{~cm}^{2}\right]$} \\
\hline \hline 3.6 & 0.02 & 0.0081 \\
\hline 3.8 & 0.048 & 0.044 \\
\hline 4 & 0.17 & 0.14 \\
\hline 4.2 & 0.44 & 0.42 \\
\hline 4.4 & 1.1 & 0.99 \\
\hline 4.6 & 2.2 & 2.1 \\
\hline 4.8 & 4 & 4.1 \\
\hline 5 & 6.9 & 6.5 \\
\hline 5.2 & 9.7 & 9.5 \\
\hline 5.4 & 13 & 13 \\
\hline 5.6 & 16 & 15 \\
\hline 5.8 & 18 & 18 \\
\hline 6 & 19 & 19 \\
\hline 6.2 & 20 & 19 \\
\hline 6.4 & 19 & 18 \\
\hline 6.6 & 16 & 16 \\
\hline 6.8 & 14 & 15 \\
\hline 7 & 12 & 12 \\
\hline 7.2 & 9.5 & 9.9 \\
\hline 7.4 & 7.5 & 7.3 \\
\hline 7.6 & 5.3 & 5.6 \\
\hline 7.8 & 3.5 & 3.9 \\
\hline
\end{tabular}

TABLE XIII: The angle-averaged neutrino effective area as a function of energy for events in the final sample satisfying $N_{\mathrm{ch}} \geq 100$. 


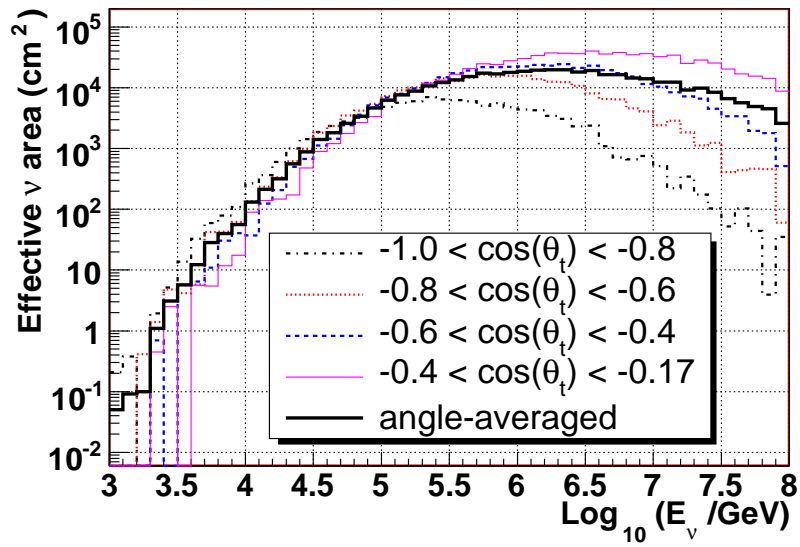

FIG. 13: Effective area for $\nu_{\mu}$ as a function of the true simulated energy at the Earth's surface in intervals of cosine of the true zenith angle, $\theta_{\mathrm{t}}$. The angle-averaged effective area is represented by the solid black line. This calculation was based on the final event sample for events satisfying $N_{\text {ch }} \geq 100$. 